Negative selection The intrathymic elimination of $\mathrm{CD} 4^{+} \mathrm{CD} 8+$ thymocytes that express $\mathrm{T}$ cell receptors with high affinity for self antigens.
Molecular Oncology Program, DeWitt Daughtry Family Department of Surgery and Sylvester Comprehensive Cancer Center, University of Miami Miller School of Medicine, Florida 33136, USA Correspondence to A.J.C. e-mail:tcapobianco@med. miami.edu doi:10.1038/nrc3035

Published online 14 April 2011

\title{
Notch signalling in solid tumours: a little bit of everything but not all the time
}

\section{Prathibha Ranganathan, Kelly L. Weaver and Anthony J. Capobianco}

\begin{abstract}
The discovery of Notch in Drosophila melanogaster nearly a century ago opened the door to an ever-widening understanding of cellular processes that are controlled or influenced by Notch signalling. As would be expected with such a pleiotropic pathway, the deregulation of Notch signalling leads to several pathological conditions, including cancer. A role for Notch is well established in haematological malignancies, and more recent studies have provided evidence for the importance of Notch activity in solid tumours. As it is thought to act as an oncogene in some cancers but as a tumour suppressor in others, the role of Notch in solid tumours seems to be highly context dependent.
\end{abstract}

A role for NOTCH1 in human cancer was originally suggested owing to a chromosomal translocation that was found in a patient with $\mathrm{T}$ cell acute lymphoblastic leukaemia (T-ALL) ${ }^{1}$. Although this translocation is rare in patients with T-ALL, it was later discovered that most T-ALL cases harbour activating mutations in the NOTCH1 locus ${ }^{2}$ (BOX 1). These mutations generally result in ligand-independent proteolytic cleavage of NOTCH1 (REF. 3) and increased stability of the active Notch intracellular domain (NICD), the net result being the constitutive activation of the Notch pathway and the neoplastic transformation of $\mathrm{T}$ cells.

Although a causative role for Notch signalling is well established in T-ALL, a uniform model for the role of Notch signalling in tumorigenesis remains elusive. Despite the wealth of data suggesting a role for Notch in solid tumours, there is little evidence to support a causative role for Notch in the initiation of tumorigenesis in human solid cancers. Indeed, unlike in T-ALL, there is little evidence for genetic alterations in Notch genes in solid tumours. But in many solid tumours, including cancers of the breast, colon, pancreas, prostate and central nervous system, Notch signalling seems to be crucial (TABLE 1; see Supplementary information S1 (table)). Interestingly, Notch signalling also seems to have a contradictory tumour suppressor role in mouse keratinocytes, pancreatic and hepatocellular carcinoma, and small-cell lung cancer (reviewed in REF. 4). Taken together, these observations indicate that Notch is exerting its effects in solid tumours owing to the aberrant activation of the pathway. Moreover, the cellular interpretation and outcome of this aberrant Notch activity is highly dependent on contextual cues such as interactions with the tumour microenvironment and crosstalk with other signalling pathways.

What accounts for the lack of observed mutations in Notch genes in solid tumours? Insight can be derived from the T-ALL paradigm. During early T cell development, mutations in NOTCH1 that result in constitutive activation can provide a cell survival advantage by bypassing the usual requirement for cell-to-cell engagement and so activating Notch signalling in order to evade negative selection. This provides a basis for the hypothesis that a cell in an epithelium cannot escape cell-to-cell contact, and so a wealth of opportunity exists for liganddependent activation of Notch signalling, making activating mutations of Notch genes less important. Therefore, in solid tumours the issue could be less one of 'constitutive' activation and more one of 'inappropriate' activation of Notch. Moreover, evidence that has been derived from studies of pancreatic cancer suggests that Notch signalling during the initial stages of tumorigenesis can prevent tumour formation, in contrast to later stages of tumour development, in which Notch activation is required $^{5,6}$. This suggests the importance of the temporal and spatial context of Notch activity. Inappropriate activation of Notch signalling in tumorigenesis can be initiated in different ways, such as through the loss of a negative regulator or the deregulated expression of the Notch receptor and ligands, as has been reported in several solid tumours, including prostate tumours ${ }^{7}$, pancreatic tumours ${ }^{8}$, glioblastoma $a^{9}$ and breast tumours ${ }^{10}$. 


\section{At a glance}

- A causative role for Notch signalling is well established in T cell acute lymphoblastic leukaemias (T-ALLs), which have activating mutations in the Notch genes resulting in a constitutively active pathway. By contrast, solid tumours, which have ample opportunity to activate the pathway, exhibit inappropriate activation by multiple mechanisms, such as overexpression of ligand or loss of negative regulators of the pathway.

- The role of Notch signalling in solid tumours is highly dependent on the spatial and temporal context of Notch activation, as well as the status of other signalling pathways in the cells.

- Notch signalling has opposing roles in tumorigenesis depending on the cell type. Opposite interactions of the Notch pathway have been documented with the WNT and $\mathrm{p} 53$ pathways. Although synergy with WNT and antagonism of the $\mathrm{p} 53$ pathway directs the oncogenic role of Notch, the opposite is seen in the tumour suppressor context.

- Notch signalling has a major role in the maintenance and progression of tumours by promoting epithelial to mesenchymal transition (EMT) and angiogenesis. It also confers resistance to radiation and chemotherapeutic agents.

- The knowledge of the extensive crosstalk of the Notch pathway with other pathways such as the epidermal growth factor receptor (EGFR) pathway could prove useful in developing combinatorial cancer therapies. receptors, the DSL proteins are type I transmembrane proteins. On binding to the Notch receptor, the ligand induces a conformational change, exposing the S2 cleavage site in the ECD to the metalloproteinase tumour necrosis factor- $\alpha$-converting enzyme (TACE; also known as ADAM17). Following S2 cleavage, Notch undergoes a third cleavage (S3 cleavage) that is mediated by the presenilin- $\gamma$-secretase complex, which is composed of presenilin 1 (PSEN1), PSEN2, nicastrin (NCSTN), presenilin enhancer 2 (PEN2) and anterior pharynx-defective 1 (APH1). The S3 cleavage results in the release of the active NICD from the plasma membrane and its subsequent translocation into the nucleus ${ }^{14}$. It is the S3 cleavage that is targeted by the class of compounds known as $\gamma$-secretase inhibitors (GSIs). Therefore, treatment with GSIs blocks the terminal cleavage and release from the plasma membrane, preventing Notch signalling. Once in the nucleus, Notch concomitantly mediates the conversion of the CBF1$\mathrm{Su}(\mathrm{H})$-LAG1 (CSL) repressor complex into a transcriptional activation complex and the recruitment of the co-activator protein mastermind-like 1 (MAML1) ${ }^{15}$. Notch signalling is thought to exert its pleiotropic effects by initiating a transcriptional cascade that involves both the activation and the repression of target genes, including transcriptional regulation by epigenetic mechanisms (BOX 2). Although the details of such a transcriptional cascade are not completely realized, several well-characterized target genes have been described. Among these genes are the basic-helix-loop-helix (bHLH) transcriptional repressors hairy enhancer of split (HES) family, the hairy-related transcription factor (HRT; also known as HEY) family, Notch receptors, Notch ligands, cyclin D1 (CCND1) and MYC. Notch transcriptional activity is terminated by phosphorylation of Notch on the C-terminal PEST domain, which targets it for ubiquitylation by ubiquitin ligases, such as FBXW7 (also known as SEL10), and subsequent degradation by the proteasome (reviewed in REF. 16) (FIG. 2a). In addition, Notch signalling can be regulated by post-translational modifications on Notch or DSL proteins. Some of these factors are also deregulated in cancer (BOX 3).

Although the primary role for the DSL ligands is to initiate Notch signalling by triggering the proteolytic cascade of Notch receptors and the release of the active NICD, Notch ligands can also have distinct Notchindependent functions. Evidence suggests that DSL proteins can also undergo proteolytic cleavage, leading to the initiation of signalling events in the ligandexpressing cell ${ }^{17-21}$ (FIG. 2b). The observation that ectopic expression of JAG1 can transform rat kidney epithelial (RKE) cells independently of Notch signalling, as well as the requirement for an intact PDZ-ligand motif in JAG1, prompted the hypothesis that the Notch-DSL pathway is in fact bidirectional ${ }^{22}$. In addition, it has been observed that Notch ligands undergo processing that is similar to Notch processing - and which uses the same proteolytic machinery - and results in the release of the $\mathrm{ICD}^{17,18}$. The jagged ICD (JICD) has been shown to activate AP1-mediated transcription, which is antagonized 
by the NICD ${ }^{17}$. In many cultured cells, the ICD of the Delta-like ligand can induce growth arrest and senescence through the induction of p21 expression, and this can be overcome by the NICD. Thus, independent effects of the Delta ICD (DICD) also seem likely ${ }^{23}$ (FIG. 2b). Although Notch-independent DSL signalling events have been reported, the physiological relevance of such signalling and its role in tumorigenesis remain to be determined.

\section{Role of Notch in tumorigenesis}

Oncogene or tumour suppressor gene? The initial evidence for the oncogenic role of Notch proteins in the transformation of epithelial cells came from mouse mammary tumour virus (MMTV)-mediated insertional mutagenesis studies ${ }^{24,25}$. Retroviral activation of Int3 (now known as Notch4) by MMTV led to mammary tumorigenesis in infected mice. Furthermore, NOTCH4 was able to transform immortalized mammary epithelial cells in culture and drove mammary tumorigenesis in transgenic mice ${ }^{25,26}$. Similarly, it was shown that NOTCH1 and NOTCH2 could transform primary rodent epithelial cells in cooperation with adenoviral $\mathrm{E} \mathrm{A}^{27}$. More recent studies using models of T-ALL have demonstrated that Notch drives tumorigenesis mostly by promoting cell cycle progression and inhibiting apoptosis (reviewed in REF. 28). Consistent with our understanding of Notch signalling, these effects are thought to be the result of the transcriptional regulation of key components of the cell cycle and the tumour surveillance machinery. In contrast to these oncogenic activities, studies also suggest that Notch signalling has a tumour suppressor function in some cell types. This tumour suppressor activity is generally thought to be a result of crosstalk with other signalling pathways that govern decreased cell proliferation, increased apoptosis or the promotion of cellular differentiation. The following sections outline the various oncogenic and tumour suppressor roles of Notch in solid tumours (FIG. 3; see Supplementary information S1 (table)).

Cell cycle regulation. The first evidence that Notch signalling directly influences the cell cycle came from transformation studies on E1A immortalized RKE cells ${ }^{27,29}$. In these studies, Notch directly induced CCND1 expression and cyclin-dependent kinase 2

\section{Box 1 | Genetic alterations that affect the activity of Notch}

The first genetic alteration that identified a role for Notch in T cell acute lymphoblastic leukaemia (T-ALL) was the chromosomal translocation $\mathrm{t}(7 ; 9)(\mathrm{q} 34 ; \mathrm{q} 34.3)$, which results in the constitutive expression of the intracellular domain of NOTCH1, leading to cell proliferation and the formation of lymphoma ${ }^{1}$. Another translocation affecting Notch signalling is $t(11 ; 19)(q 21 ; p 13)$, which results in the formation of a fusion gene between mucoepidermoid carcinoma translocated 1 (MECT1) and mastermind-like 2 (MAML2), which are located at chromosomes 19p13 and 11q21, respectively. The MECT1-MAML2 fusion protein can activate Notch target genes independently of ligand stimulation ${ }^{150}$ and can also activate cyclic AMP (cAMP)-responsive genes independently of any external stimulus ${ }^{151}$. This chromosomal abnormality is seen in mucoepidermoid cancer in the salivary gland ${ }^{150}$, bronchopulmonary mucoepidermoid carcinoma ${ }^{151}$, cervical mucoepidermoid carcinoma ${ }^{152}$ and clear cell hidradenoma of the skin ${ }^{153}$.
(CDK2) activity. Further studies on mammary tumorigenesis supported this work by showing that Notch promotes transformation by inducing CCND1 expres$\operatorname{sion}^{30}$. Increased levels of JAG1, which commonly occur in breast cancers, also promote cell cycle progression by inducing CCND1 through Notch signalling ${ }^{31}$. Interestingly, Notch overexpression failed to induce T-ALL in mice that were homozygous-null for Ccnd3, which is also a target of $\mathrm{Notch}^{32}$. Although this suggests an obligatory role for D-type cyclins in Notch-mediated transformation, $\mathrm{Ccnd} 3$ probably has a broader role in tumorigenesis. MYC, a potent driver of cell cycle entry, is a direct transcriptional target of Notch and contributes to cell cycle progression in T-ALL ${ }^{33,34}$, as well as in Notch-induced mouse mammary tumours ${ }^{35}$. NOTCH1 and MYC probably control two transcriptional programmes that together regulate the growth of primary T-ALL cells ${ }^{35,36}$. Although the major mechanism by which Notch promotes cell cycle progression is through the induction of CCND1 and MYC, the inhibition of cyclin-dependent kinase inhibitors (CDKIs) also has an important role. Notch mediates the transcriptional repression of the CDKIs p27 and p57 through HES1 in different cell types ${ }^{37-39}$. In T-ALL, Notch directs the transcription of the E3 ubiquitin ligase $S$ phase kinaseassociated protein 2 (SKP2), which leads to decreased p27 protein levels and increased cell proliferation ${ }^{40}$.

Notch signalling can cooperate with other oncogenic signalling pathways. In breast epithelial cells, cooperation between Notch and RAS has been shown to exert proliferative effects and cause malignant transformation $^{41}$; however, the exact nature of this cooperation is not clear. In astrocytic gliomas, Notch signalling has an oncogenic effect owing to crosstalk with the EGF receptor (EGFR) pathways and the subsequent activation of the PI3K-AKT pathway, KRAS, CCND1 and matrix metalloproteinase 9 (MMP9) ${ }^{42}$. Interaction between Notch and the JAK-signal transducer and activator of transcription (STAT) pathway also leads to a proliferative response, which may initiate tumour growth. In developmental systems such as D. melanogaster, crosstalk between Notch signalling and the JAK-STAT pathway is responsible for maintaining the balance between intestinal stem cell self-renewal and differentiation ${ }^{43}$, and this mechanism may also be at work in malignant cells.

By contrast, the activation of EGFR signalling has been associated with the loss of Notch expression. Inhibition of $\gamma$-secretase can result in increased EGFR signalling and the subsequent proliferation of cells ${ }^{44}$. Active Notch signalling, coupled with the inhibition of multiple pathways that are mainly downstream of receptor tyrosine kinases (RTKs) ${ }^{45-48}$, can decrease tumour cell proliferation $^{45-49}$. In prostate cancer cells, which often have low levels of the tumour suppressor PTEN, ectopic activation of Notch inhibits proliferation concomitantly with an increase in the levels of PTEN, suggesting that PTEN is under the control of Notch ${ }^{50,51}$. However, it is not yet known how Notch regulates the expression of PTEN to inhibit tumour formation while also inducing epithelial to mesenchymal transition (EMT) and cellular invasion ${ }^{52}$. In human and mouse epithelial cell 
Table 1 | Multiple roles of Notch signalling in solid tumours*

\begin{tabular}{|c|c|c|c|c|c|}
\hline Tumour type & Oncogenic & $\begin{array}{l}\text { Tumour } \\
\text { suppressor }\end{array}$ & $\begin{array}{l}\text { Tumour } \\
\text { progression }\end{array}$ & $\begin{array}{l}\text { Tumour } \\
\text { maintenance }\end{array}$ & Drug resistance \\
\hline Breast & $\checkmark$ & $\checkmark$ & $\checkmark$ & $\checkmark$ & $\checkmark$ \\
\hline Colorectal & $\checkmark$ & & $\checkmark$ & & $\checkmark$ \\
\hline Prostate & & $\checkmark$ & $\checkmark$ & & \\
\hline Liver & & $\checkmark$ & $\checkmark$ & & $\checkmark$ \\
\hline Pancreatic & $\checkmark$ & & $\checkmark$ & & $\checkmark$ \\
\hline Glioblastoma & & $\checkmark$ & $\checkmark$ & $\checkmark$ & $\checkmark$ \\
\hline Cervical & $\checkmark$ & & $\checkmark$ & & $\checkmark$ \\
\hline Oral SCC & $\checkmark$ & $\checkmark$ & & & \\
\hline Skin & & $\checkmark$ & & & \\
\hline Head and neck & & & & & $\checkmark$ \\
\hline Medulloblastoma & & & $\checkmark$ & $\checkmark$ & \\
\hline Melanoma & & & $\checkmark$ & $\checkmark$ & \\
\hline Lung & $\checkmark$ & $\checkmark$ & $\checkmark$ & & \\
\hline
\end{tabular}

lines, Notch activity, together with transforming growth factor- $\beta$ (TGF $\beta$ ) signalling, can cause cell cycle arrest. TGF $\beta$ signalling leads to an induction in the expression levels of p21 and JAG1. The increased levels of JAG1 activate Notch signalling, which sustains the levels of p21, resulting in cell cycle arrest ${ }^{53}$ (FIG. 3). However, the opposite relationship between Notch and TGF $\beta$ signalling has been observed in breast and cervical cancer cells. Breast cancer cells that express the NOTCH4 ICD are resistant to TGF $\beta$-mediated growth arrest, but treating these cells with GSIs can resensitize them ${ }^{54}$. In cervical cancer cells, NOTCH1 signalling confers resistance to the growth inhibitory effects of TGF $\beta^{55}$. These opposing actions of Notch and TGF $\beta$ crosstalk seem to be both cell type specific and Notch paralogue dependent.

It is likely that a complex combination of factors determines the pro-tumorigenic or antitumorigenic effects of Notch crosstalk, including multiple interactions with the tumour microenvironment. For example, Notch signalling has a tumour suppressor effect in skin epithelial cells. Loss of Notch1 in epidermal keratinocytes impairs skin barrier integrity and creates a wound-like niche that promotes tumorigenesis in a non-cell autonomous manner. Using a chimeric mouse model, it was demonstrated that in such a tumour-promoting microenvironment, expression of NOTCH1 in keratinocytes was insufficient to suppress this tumour-promoting effect, emphasizing the importance of crosstalk between this barrier-defective epidermis and its stroma ${ }^{56}$. It has also been demonstrated that loss of Notch signalling in the skin leads to improper epidermal differentiation and a defective skin barrier, resulting in inflammation and lymphoproliferative and myeloproliferative disorders ${ }^{57,58}$. This emphasizes that Notch signalling in the microenvironment can have a tumour suppressive effect.
Inhibition of apoptosis. Inhibition of apoptosis is an essential step in tumorigenesis. One of the key mechanisms by which Notch inhibits apoptosis is through the negative regulation of $\mathrm{p} 53$ and PTEN. Contrary to the positive regulation of PTEN by Notch in prostate cancer cells, the inhibition of Notch by GSIs in T-ALL cells increases PTEN expression. This is probably due to the decreased expression of HES1, which is a negative regulator of $\mathrm{PTEN}^{59}$. Decreased PTEN activity results in the activation of PI3K-AKT signalling through mTOR, which leads to the phosphorylation of MDM2 and culminates in the inhibition of p53 (REF. 60). In breast epithelial cells, the expression of active Notch results in the activation of the PI3K-AKT pathway by an autocrine loop, and so prevents apoptosis ${ }^{61}$. However, the activation of PI3K-AKT pathway is not accompanied by the downregulation of PTEN, suggesting that the repression of PTEN by Notch (via HES1) is highly context dependent ${ }^{61,62}$. Ectopic expression of NOTCH1 can also inhibit p53 activity by blocking its nuclear translocation or by preventing the serine phosphorylation that is necessary for p53 activation ${ }^{63}$. In T-ALL, Notch seems to disrupt the ARF-MDM2-p53 tumour surveillance pathway through the repression of ARF expression ${ }^{64}$, which results in decreased apoptosis. A similar mechanism in solid tumours has not yet been described.

By contrast, evidence suggests that Notch signalling can induce apoptosis by increasing p53 activity in some cell types (reviewed in REF. 49). In human keratinocyte tumours, studies have shown that NOTCH1 expression is under the direct transcriptional control of p53 (REF. 45). In hepatocellular carcinoma, ectopic expression of NOTCH1 increases the sensitivity of cancer cells to p53-mediated apoptosis by reducing proteasomal degradation of p53 by the AKT-MDM2 pathway. This in turn induces the expression of death receptor 5 


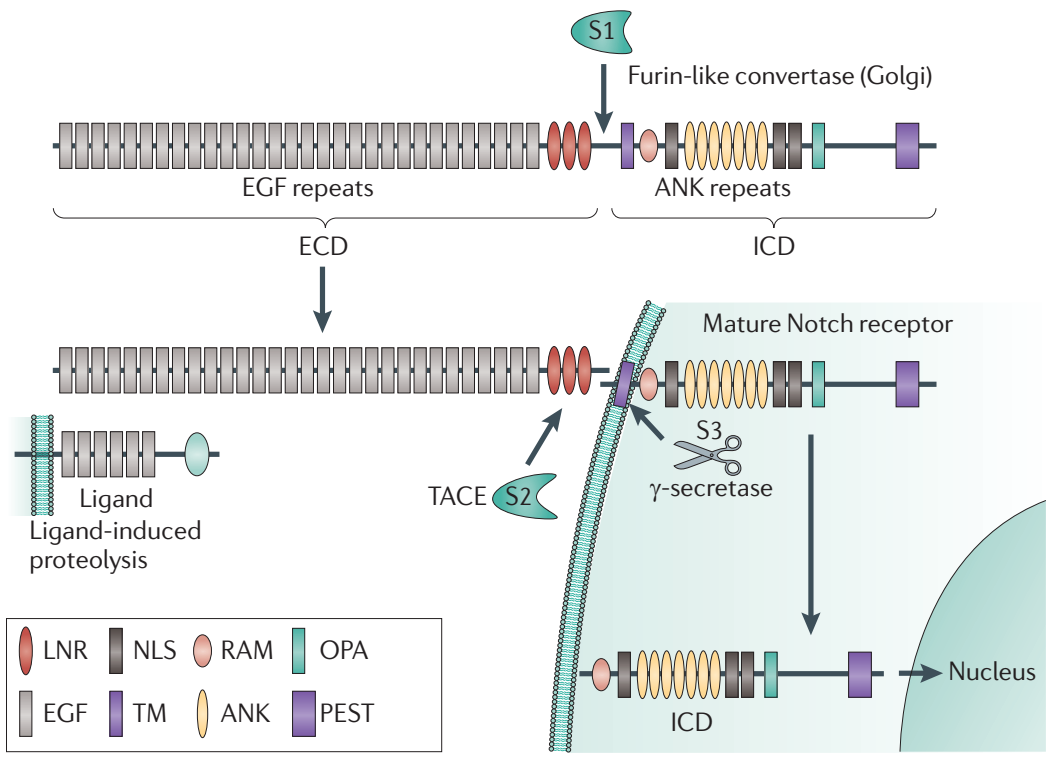

Figure 1 | Structural organization and proteolytic processing of the Notch receptor. Notch proteins are synthesized as precursor forms that are cleaved by furin-like convertase ( 1 1 cleavage) to generate the mature receptor, which is composed of two subunits that are held together by non-covalent interactions. The extracellular domain (ECD) of the Notch protein is comprised of epidermal growth factor (EGF)-like repeats, three cysteine-rich LIN12 and Notch repeats (LNRs), followed by a carboxy-terminal hydrophobic region. The Notch intracellular domain (NICD) is composed of conserved protein domains: namely, the RBP-JK-associated module (RAM) domain, ankyrin (ANK) repeats, nuclear localization signals (NLSs) and the PEST domain. The general domain organization of the Notch proteins, with the details of NOTCH1, is shown. However, there are differences observed among the four receptors (reviewed in REF. 14). On binding to the Notch receptor, the ligand induces a conformational change, exposing the S2 cleavage site in the ECD to the metalloproteinase tumour necrosis factor- $\alpha$-converting enzyme (TACE; also known as ADAM17). Following S2 cleavage, Notch undergoes a third cleavage (S3) that is mediated by the presenilin- $\gamma$-secretase complex, which is composed of presenilin 1 (PSEN1), PSEN2, nicastrin (NCSTN), presenilin enhancer 2 (PEN2) and anterior pharynx-defective 1 (APH1). The $\mathrm{S} 3$ cleavage results in the release of the active $\mathrm{NICD}$ from the plasma membrane and the subsequent translocation into the nucleus. ICD, intracellular domain; OPA, polyglutamine repeat-containing region; TM, transmembrane.

(DR5; also known as TNFRSF10B), resulting in cell death and the inhibition of tumour formation ${ }^{46}$. It is possible that p53 is activated merely as a cellular response to Notch-induced proliferation, which is analogous to the effect of other oncogenes such as mutant RAS or E1A.

There are also examples from studies on cervical cancer and Ewing's sarcoma in which Notch activates p53 (reviewed in REF. 49). In some human papilloma virus (HPV)-positive cervical cancer cell lines (such as HeLa), ectopic expression of the NICD results in the downregulation of HPV E6 and E7 transcription by decreasing AP1 activity, leading to the activation of $\mathrm{p} 53$, the inhibition of $\mathrm{RB}$ hyperphosphorylation and growth arrest ${ }^{47,48}$. Conversely, Notch inhibits apoptosis in cervical cancer cells through the activation of nuclear factor- $\kappa \mathrm{B}(\mathrm{NF}-\kappa \mathrm{B})^{65,66}$. Studies in human and mouse T-ALL, and in other cell types, have shown that Notch induces the transcription of NF- $\kappa B$ pathway components, which may operate as a feedforward activation of NF- $\mathrm{kB}$ activity. A physical interaction between the NICD and the inhibitor of NF- $\mathrm{KB}$ kinase (IKK) complex has also been described, resulting in the activation of NF-kB (reviewed in REF. 67).
Reprogramming of differentiation. A balance between the proliferation of undifferentiated cells and their differentiation into mature cell types is key to maintaining tissue homeostasis. Under normal conditions, the programmes that govern differentiation and proliferation are tightly regulated by many 'cues' in the cellular milieu. Signalling pathways, such as those triggered by growth factors, Notch, WNT and Hedgehog (HH), act together to coordinately regulate these events. Inappropriate activation of any of these pathways can result in deregulated proliferation and differentiation programmes that lead to tumorigenesis. Crosstalk between Notch signalling and WNT signalling has been shown to initiate tumorigenesis mainly by disrupting the balance between progenitor cell proliferation and differentiation, thus maintaining cells in an undifferentiated state ${ }^{68}$. The WNT pathway can be activated in a number of ways, including through the constitutive activation of $\beta$-catenin owing to mutations in adenomatous polyposis coli (APC) or AXIN $^{69-72}$; the silencing of genes that express inhibitory WNT ligands $s^{73,74}$; the overexpression of WNT receptor or ligands $s^{75-78}$; and the activating mutations in low-density lipoprotein receptorrelated protein $5(\mathrm{LRP} 5)^{79}$. For example, Apc-mutant mice develop multiple intestinal tumours owing to the constitutive activation of $\beta$-catenin. Blocking Notch signalling in these mice by GSI treatment results in the differentiation of the proliferative cells into more differentiated goblet cells, suggesting that Notch signalling might have a role in inhibiting differentiation and therefore may play a part in $\beta$-catenin-driven tumorigenesis ${ }^{80}$. Several lines of evidence suggest that Notch and WNT interact genetically, and there are direct physical associations between components of each pathway ${ }^{81-84}$. For example, $\beta$-catenin has been shown to directly bind the NICD, resulting in an increased transcriptional output of target genes ${ }^{84}$. In addition, MAML1 has been reported to function as a coactivator for $\beta$-catenin-dependent transcription ${ }^{85}$, raising the possibility that signalling pathways can converge through common components.

In the skin, however, Notch suppresses tumorigenesis by blocking WNT signalling, thereby driving cells towards a more differentiated phenotype. In keratinocytes, WNT- $\beta$-catenin signalling has been associated with malignancies and with the maintenance of multipotent stem cell populations, so it is possible that the inhibition of the WNT pathway is sufficient to drive these cells towards a more differentiated phenotype. NOTCH1 activation in keratinocytes results in the repression of $\beta$-catenin signalling. Deletion of Notch1 in the mouse epidermis results in inappropriate activation of $\beta$-catenin, and the formation of skin tumours ${ }^{86}$. Notch can also downregulate the expression of the WNT ligands Wnt3 and Wnt 4 through HES1 and p21 (REF. 87), providing further mechanisms through which Notch can suppress tumorigenesis by inhibiting the WNT pathway. Although assiduously investigated, the mechanism of crosstalk between these two pathways and their interactions in tumorigenesis remain unclear (FIG. 3).

Other pathways may also crosstalk with Notch to block differentiation and to drive tumorigenesis. In pancreatic adenocarcinoma, interaction between Notch 
Exocrine pancreas

The portion of the pancreas that secretes digestive

enzymes that are then passed on to the small intestine. and RAS-MAPK signalling has been implicated in the initiation of tumours. NOTCH1 is induced by KRAS signalling, and this results in dedifferentiation or in the inhibition of differentiation in the exocrine pancreas, leading to the formation of pancreatic intraepithelial neoplasia (PanIN) ${ }^{88,89}$. These lesions accumulate further genetic alterations and form aggressive pancreatic ductal adenocarcinoma (PDAC) ${ }^{88,89}$. Interestingly, it has been hypothesized that under physiological conditions Notch can act as a negative regulator of RAS signalling and can induce the differentiation of several pancreatic cell types ${ }^{90}$, thereby creating a context in which Notch functions as a tumour suppressor. This is supported by a recent study that demonstrated that Notch can function as a tumour suppressor in pancreatic cancers, in which deleting Notch1 in the context of activated KRAS resulted in enhanced tumour formation in mouse mod$\mathrm{els}^{5}$. These studies underscore the hypothesis that the outcome of Notch signalling in tumorigenesis mostly depends on the temporal and spatial context in a given tissue.

\section{Notch in tumour progression}

As well as influencing tumour initiation, Notch is also important for aspects of tumour progression, including angiogenesis, EMT-driven metastatic growth and the maintenance of cancer stem cells.

Regulation of angiogenesis. Notch receptors and ligands are widely expressed in the vasculature, suggesting the importance of the Notch signalling pathway in angiogenesis. During normal angiogenesis, vascular endothelial growth factor (VEGF) drives the budding of new vessels by increasing the number of DLL4-expressing tip cells that bud out of a pre-existing

\section{Box 2 | Notch and epigenetic regulation in Drosophila melanogaster}

Epigenetic regulation of cancer has gained considerable importance over the past few years. The reversibility of these changes, unlike genetic alterations, makes them promising targets for therapy. Epigenetic silencing of the Notch locus by histone methylation from Polycomb group ( $\mathrm{PcG}$ ) proteins is a mechanism through which the activity of Notch is kept under check in the Drosophila melanogaster eye ${ }^{154}$. Although evidence is very limited, there are also indications of epigenetic silencing that is mediated by Notch at its target loci. Using the $D$. melanogaster eye as a model system, Ferres-Marco et al. ${ }^{155}$ showed that Notch activation cooperates with the overexpression of the Polycomb epigenetic silencers Pipsqueak and Lola in tumorigenesis. Collectively, these events result in the silencing of genes such as Retinoblastoma-family protein (Rbf), resulting in the formation of metastatic tumours. This began to unravel the crosstalk between the Notch pathway and the epigenetic pathways in growth control and tumorigenesis. However, whether Notch activation can directly modulate the expression and activity of these epigenetic silencers is yet to be established.

Providing an additional link between Notch signalling and epigenetic regulation, the repression of Notch target genes during $D$. melanogaster development is caused by modulating the chromatin structure, probably through histone chaperones. ASF1 is a histone chaperone that has been found to bind and inactivate Notch target loci by interacting with $\mathrm{Su}(\mathrm{H}) / \mathrm{H}$ (the $\mathrm{D}$. melanogaster homologue of the mammalian $\mathrm{CSL}$ complex) and removing $\mathrm{H} 3 \mathrm{~K} 4 \mathrm{me} 3$ (REF. 156). It is unclear whether ASF1 can target all loci that contain binding sites for $\mathrm{Su}(\mathrm{H}) / \mathrm{H}^{157}$ or whether it may be a target of Notch signalling, thus resulting in negative feedback regulation. Identification of a similar mechanism in cancer would greatly aid the development of a strategy to disrupt Notch activity at the transcriptional level. vesse ${ }^{91}$. Although these endothelial cells are nonproliferative, they are followed by several motile, proliferative endothelial tube cells, which express Notch and form the lumen of the new vessel. DLL4 on the tip cells signals through Notch on the adjacent tube cells to decrease VEGF-induced sprouting and branching by downregulating VEGF receptor 2 (VEGFR2) ${ }^{92,93}$. In this manner, DLL4 inhibits angiogenesis by a negative feedback loop with VEGF (FIG. 4).

In the hypoxic tumour environment, tumour cells secrete large amounts of VEGF, which results in the expression of comparatively higher levels of DLL4 by endothelial cells in the stroma ${ }^{94,95}$. Subsequently blocking VEGF activity in such tumours resulted in decreased DLL4 expression in tumour endothelial cells $^{96,97}$. The close relationship between VEGF and DLL4 expression led to the examination of the effect of blocking DLL4-mediated Notch signalling on adjacent endothelial cells, which resulted in a substantial reduction in tumour growth. Surprisingly, this was associated with an increase in vessel formation ${ }^{98}$, possibly because DLL4 is the factor responsible for the downregulation of VEGF-induced angiogenesis. This vasculature was nonfunctional, suggesting that DLL4-Notch is responsible for some specialized functions in the vessels that form in response to VEGF, such as the development of the vessel lumen ${ }^{98}$. These results suggest that in the future it could be useful to combine VEGF inhibitors and Notch signalling inhibitors in anti-angiogenic therapy (reviewed in REF. 96).

DLL4 and JAG1 have distinct roles during angiogenesis, and they maintain a balance between endothelial cell sprouting and the formation of new vessels. Spatiotemporal regulation of Notch activation during this process is brought about by Fringe proteins ${ }^{99}$. This family of $\mathrm{N}$-acetylglucosaminidyl transferases (comprised of lunatic fringe (LFNG), radical fringe (RFNG) and manic fringe (MFNG)) modulates the activity of Notch proteins through the glycosylation of the EGF-like repeats. Studies from D. melanogaster indicate that the Fringe proteins inhibit Serrate (D. melanogaster jagged homologue)-dependent Notch activation and potentiate Delta-dependent Notch activation $^{100}$ (FIG. 2a). This mechanism might also operate in other Notch-controlled biological processes, such as cancer progression and tumour angiogenesis.

Endothelial cell migration is an essential step in the production of new blood vessels. Studies in developmental systems demonstrate that the TGF $\beta$ and bone morphogenetic protein (BMP) pathways interact with the Notch pathway through SMADs, leading to alterations in endothelial cell migration. Although there is little evidence for an interaction between Notch and TGF $\beta$ in tumour angiogenesis, studies in developmental model systems suggest that a mechanism through which Notch may promote tumour growth is the repression of TGF $\beta$-induced inhibition of endothelial cell growth ${ }^{101}$. In addition, some BMP family members can induce the expression of Hey 1 (also known as Herp2) synergistically with Notch. HEY 1 then negatively regulates the activity of ID1, 
a

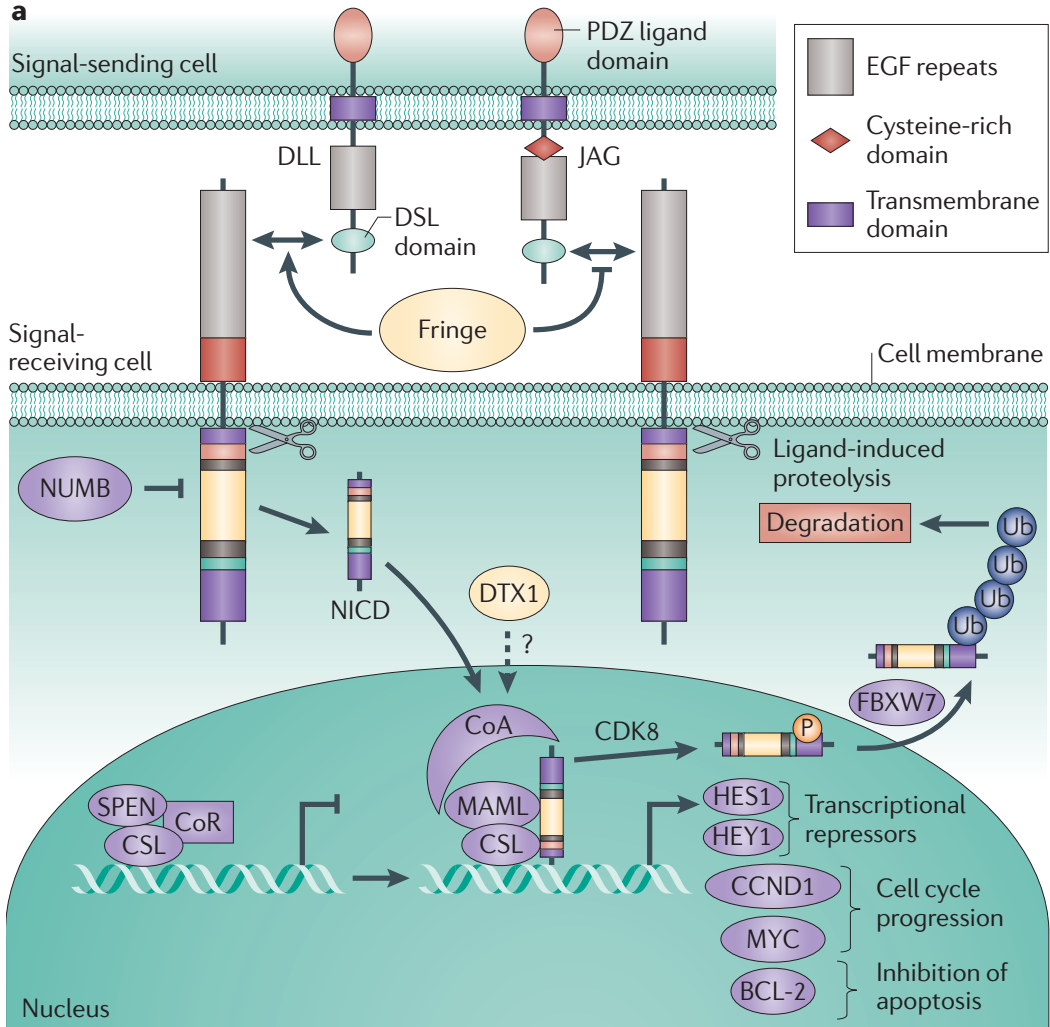

b

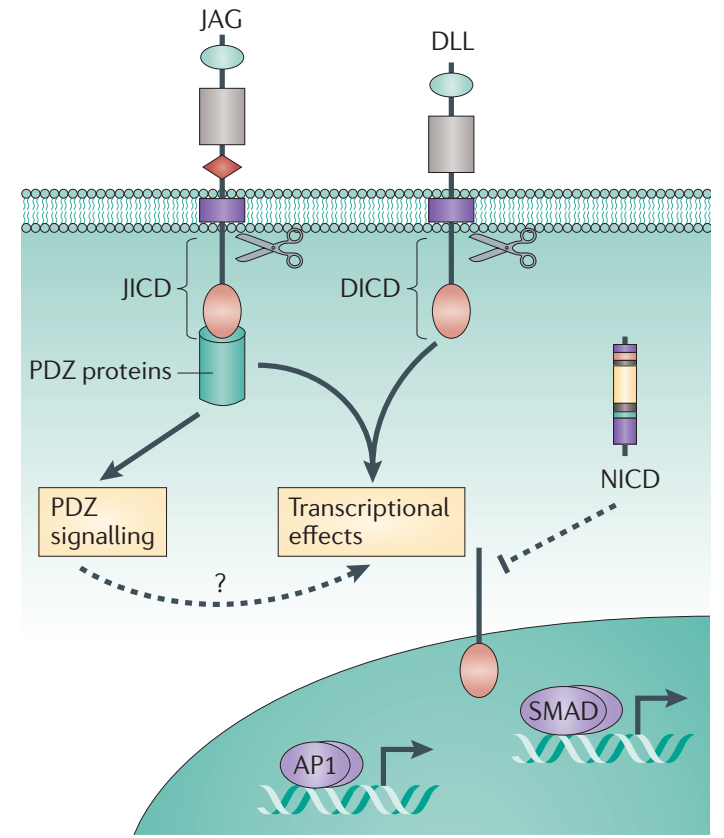

Figure 2 | Signal transduction from Notch receptors and ligands. a | Signal transduction from Notch receptors is shown. Notch signalling is activated by interaction between the ligand-expressing cell and the Notch-expressing cell, followed by proteolytic cleavage that releases the Notch intracellular domain (NICD) (FIG. 1). Before activation of Notch signalling, CBF1-Su(H)-LAG1 (CSL) is bound to DNA along with co-repressors (CoRs) such as MSX2-interacting protein (SPEN; also known as MINT and SHARP). On activation of Notch, the NICD recruits the co-activator (CoA), mastermind-like 1 (MAML1) and others, and thus converts the CSL-repressor complex into a transcriptional activator complex and drives the transcription of target genes. The signal is terminated by phosphorylation (P) of the PEST domain of the NICD, followed by ubiquitylation (Ub) by FBXW7 (also known as SEL10) and proteasomal degradation. Note that when the extracellular domain of Notch is glycosylated by Fringe proteins, the binding between Notch and Delta-like (DLL) is favoured and jagged (JAG) can no longer bind to and activate Notch. Deltex 1 (DTX1) inhibits Notch activity by preventing the recruitment of CoAs. It could also mediate CSL-independent effects of Notch. NUMB promotes ubiquitylation of the membrane-bound NOTCH1 and targets the NICD for proteasomal degradation. $\mathbf{b}$ | Signal transduction from Notch ligands is shown.

Proteolytic cleavage releases the intracellular domain (ICD) of the Notch ligands. The PDZ ligand (PDZL) domain interacts with PDZ proteins, resulting in a signalling cascade. The ICD can also enter the nucleus and regulate transcription, possibly through interactions with AP1 or the SMAD proteins. This transcriptional regulation may be antagonized by the NICD. Dashed arrows indicate poorly understood mechanisms. CCND1, cyclin D1; CDK8, cyclin-dependent kinase 8; DICD, Delta ICD; EGF, epidermal growth factor; HES1, hairy enhancer of split 1; JICD, jagged ICD.

a promoter of endothelial cell migration ${ }^{102}$. This results in the inhibition of endothelial cell migration and functions as a crucial switch downstream of the Notch and BMP pathways ${ }^{102}$.

EMT. The growth of solid tumours is highly dependent on their interaction with the microenvironment, which provides a favourable milieu for their growth and progression. These tumour-microenvironment interactions have an important role in regulating EMT (FIC. 4). The phenomenon of EMT occurs when epithelial cells undergo several morphological changes and take on a mesenchymal phenotype, including decreased adhesion, increased production of extracellular matrix components, increased migration, increased resistance to apoptosis and invasiveness. EMT is a prerequisite for the tumour cells to cross the basement membrane, enter into circulation and result in distant metastases (reviewed in REF. 103) (FIG. 4).

Recent studies have suggested that Notch can drive EMT by upregulating the expression of two target genes, SNAIL (also known as SNAI1) and SLUG (also known as $S N A I 2$ ), which are transcriptional repressors of $\mathrm{CDH1}$, the gene encoding E-cadherin. In breast cancer, JAG1 activation of Notch signalling induces EMT through the upregulation of $S L U G^{104}$. A study of 154 prostate tumour samples showed an association between high expression of JAG1 and increases in metastases and tumour recurrence ${ }^{7}$. This study also suggested that the pro-metastatic activity of JAG1 is mediated by the induction of EMT through the AKT signalling pathway $^{7}$. Notch might also synergize with hypoxia-inducible 


\section{Box 3 | Notch regulators and tumorigenesis}

Several processes, including proteolysis, glycosylation, ubiquitylation and phosphorylation, control Notch activation. Aberrant activation of the Notch pathway can be caused by the overexpression of ligands or factors that activate the receptor or by the loss of negative regulators. Some of these are deregulated in cancers, resulting in aberrant Notch signalling.

Although ubiquitylation of proteins is generally associated with degradation, it also has a role in signal transduction by facilitating receptor activation and endocytosis, as is seen in ligand-dependent Notch signalling. For example, the ubiquitin ligase skeletrophin (also known as MIB2), which ubiquitylates jagged 2 (JAG2), is overexpressed in multiple myeloma, facilitating the cleavage of NOTCH1 and activating Notch-mediated transcription in stromal cells ${ }^{158}$. In melanoma, however, the expression of skeletrophin is lost, through loss of heterozygosity (LOH), promoter methylation or downregulation by SNAIL (also known as SNAI1), thus contributing to tumour suppression ${ }^{159}$.

FBXW7 (also known as SEL10) is the substrate-recognition subunit of an E3 ubiquitin ligase complex that degrades Notch proteins ${ }^{160}$ (FIG. 2a). FBXW7 is thought to be a tumour suppressor because it is deregulated, lost or mutated in several cancers, including colorectal cancer, cholangiocarcinomas and endometrial cancers ${ }^{161,162}$. Mutations at hot spots such as Arg465 and Arg479 result in the abrogation of substrate recognition and the inappropriate stabilization of several oncoproteins, including Notch ${ }^{161}$. FBXW7 function is also compromised by the latency-associated nuclear antigen (LANA) of the Kaposi's sarcoma virus, which binds to the carboxyl terminus of FBXW7, preventing its association with the Notch intracellular domain (NICD). As a result, NICD is stabilized and has increased activity, leading to the proliferation of the virus-infected cells ${ }^{163}$.

NUMB and NUMB-like proteins function as signalling inhibitors for Notch by targeting the membrane-bound Notch for degradation following activation ${ }^{164}$. Loss of NUMB has been associated with breast carcinogenesis, and possibly results in the stabilization and hyperactivation of $\mathrm{Notch}^{165}$. In addition, NUMB binds to $\mathrm{p} 53$ and MDM2 to prevent ubiquitylation of p53. Thus, loss of NUMB in a large proportion of breast cancers can result in increased Notch activity and loss of p53 and an aggressive tumour phenotype with poor prognosis ${ }^{166}$.

Another important, but not well understood, regulator of Notch signalling is Deltex. This was originally identified as a positive regulator of Notch signalling in Drosophila melanogaster ${ }^{167}$. The human homologue DTX1 (also known as deltex 1) was subsequently identified ${ }^{168}$. Although Deltex has been demonstrated to inhibit Notch activity by preventing the recruitment of co-activators to the CBF1-Su(H)-LAG1 (CSL)-Notch-MAML complex ${ }^{169}$, it could function as a positive regulator of Notch signalling independently of CSL in some cell types ${ }^{170}$ (FIG. 2a).

factor $1 \alpha$ (HIF1A) and HIF2A to induce EMT and therefore increase metastasis. Blocking either HIF or the Notch co-activator MAML1 in breast, colon or cervical cancer cells reduced the invasion and metastatic ability of these cells ${ }^{105,106}$. Furthermore, crosstalk between Notch and TGF $\beta$ is important for the initiation of EMT, as Notch signalling is required to sustain TGF $\beta$-induced HEY1 expression ${ }^{107}$.

Although research suggests that EMT is a prerequisite for metastases, recent evidence indicates that EMT that is mediated by Notch or any other factors can give rise to a stem cell-like phenotype, including increased resistance to apoptosis and anoikis ${ }^{108}$.

Cancer stem cells. Cancer stem cells (CSCs; also known as tumour-initiating cells) were first described as a multipotent subpopulation of acute myeloid leukaemia cells ${ }^{109}$ that can self-renew symmetrically or that can divide asymmetrically to produce daughter cells that continue to proliferate and so sustain tumour growth $^{110,111}$. Recent studies have also identified CSCs in many solid tumours ${ }^{112-120}$. These cells have mostly been isolated on the basis of the expression of various cell surface markers, the relevance of which remains controversial. CSCs have been proposed to be resistant to radiation and chemotherapy, possibly owing to their elevated DNA damage response, their low proliferation rate $^{121}$ or their increased expression of $\mathrm{ABC}$ transporters ${ }^{121-123}$.

Notch regulates the self-renewal properties and differentiation states of various cell types, including stem cells. Interaction between HIF1A and Notch has been shown to have a role in maintaining neuronal precursors in an undifferentiated state, and aberrant functioning of these cells can result in the formation of medulloblastomas ${ }^{124}$. Inhibition of Notch signalling or HIF1A in these cells results in their differentiation, suggesting a role for HIF1A-induced Notch signalling in maintaining stem cell characteristics ${ }^{124,125}$. Aberrant activation of Notch signalling by a DSL peptide has been shown to increase the self-renewal capacity of normal mammary stem cells, leading to a tenfold increase in mammosphere formation ${ }^{126}$. Breast CSC populations show an upregulation of Notch gene expression, and blocking Notch activity using a GSI or a neutralizing antibody to NOTCH4 reduced the mammosphere-forming ability of these cells in culture $^{127,128}$. Likewise, brain tumour stem cells have also been shown to overexpress NOTCH1, and overexpression of NOTCH1 in human glioma cell lines increased the formation of neurospheres ${ }^{129}$. It is thought that Notch signalling in these neurospheres enhances their self-renewal capacity while inhibiting their differentiation into glial and neural progenitor cells ${ }^{130-132}$. Blocking the Notch signalling pathway with a GSI decreased the growth of neurospheres in vitro and the growth of tumour xenografts in vivo. This study also suggested that blocking Notch activity results in the decreased phosphorylation of AKT and STAT3, leading to decreased CSC proliferation and increased apoptosis $^{133}$.

A considerable body of evidence has implicated Notch signalling in many processes that are linked to the progression and maintenance of the tumour 


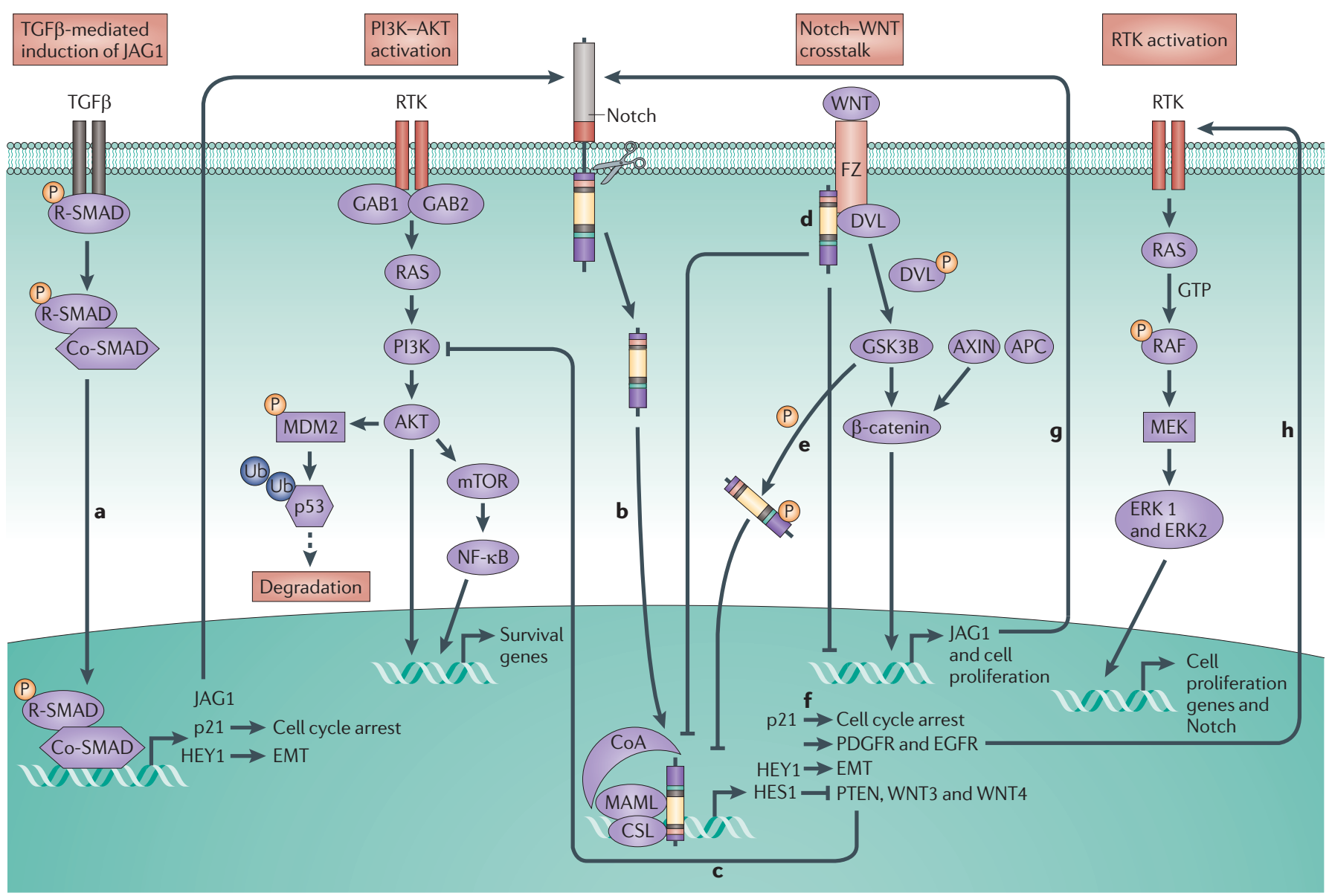

Figure 3 | Oncogenic and tumour suppressive interactions of Notch. Cleavage of the Notch intracellular domain (NICD) initiates a signalling cascade that interacts with other oncogenic and tumour suppressive pathways at multiple points. Jagged 1 (JAG1) is transcriptionally induced by the transforming growth factor- $\beta$ (TGF $\beta$ ) pathway (part a), which in turn activates Notch in an adjacent cell. Both TGF $\beta$ and Notch signalling lead to the induction of the cyclin-dependent kinase inhibitor p21, resulting in cell cycle arrest. HEY1 is another target of both pathways and is a mediator of the epithelial to mesenchymal transition (EMT). Notch signalling downregulates the expression of PTEN via the induction of hairy enhancer of split 1 (HES1) (part b), leading to the activation of the pro-survival PI3K-AKT pathway (part c). Binding of the NICD to a dishevelled protein (DVL) inhibits both the Notch and WNT pathways (part d). Phosphorylation (P) of Notch by glycogen synthase kinase $3 \beta$ (GSK3B) inhibits Notch-mediated transcription (part e). Notch signalling inhibits the WNT ligands through the induction of HES1, thereby inhibiting the tumorigenic effects of WNT signalling (part f). By contrast, JAG1 is a transcriptional target of WNT, leading to WNT-mediated activation of Notch signalling (part $\mathbf{g}$ ). Notch activates receptor tyrosine kinase (RTK) pathways by inducing the expression of the RTKs epidermal growth factor receptor (EGFR) and platelet-derived growth factor receptor (PDGFR) (part $\mathbf{h}$ ), leading to activation of cell proliferation genes, as well as positive feedback to Notch signalling. The interactions depicted in this figure are from a variety of systems. The specific interactions among the pathways are highly context dependent. APC, adenomatous polyposis coli; CoA, co-activator; Co-SMAD, common mediator SMAD; CSL, CBF1-Su(H)-LAG1; FZ, frizzled; GAB, GRB2-associated-binding protein; MAML, mastermind-like; NF- $\mathrm{B}$, nuclear factor- $\kappa B$; R-SMAD, receptor-regulated SMAD; Ub, ubiquitylation. Dashed arrow indicates a poorly understood mechanism.

phenotype. Clearly, in several distinct tumour types, abrogation of Notch signalling affects these processes and tumour growth. However, what remains unresolved is the relationship between these processes as mediated by Notch in any given tumour. For example, is the control of EMT by Notch in breast cancer linked to its role in promoting self-renewal of the CSCs and metastases? In other words, does Notch signalling alone direct these cell processes in a tumour or is the outcome of Notch signalling dependent on other crosstalk signals (FIG. 4)?

\section{Notch and drug resistance}

A major survival advantage that cancer cells can acquire is resistance to chemotherapeutic agents. This occurs mainly by activating survival pathways or by inhibiting apoptotic pathways, and Notch signalling is a major regulator of these survival pathways, through mechanisms that may be similar to its role in tumorigenesis (FIG. 3). For example, treatment of colorectal cancer with oxaliplatin activates the Notch pathway and prosurvival pathways, such as PI3K-AKT. Moreover, blocking Notch activation using GSIs sensitizes cells 


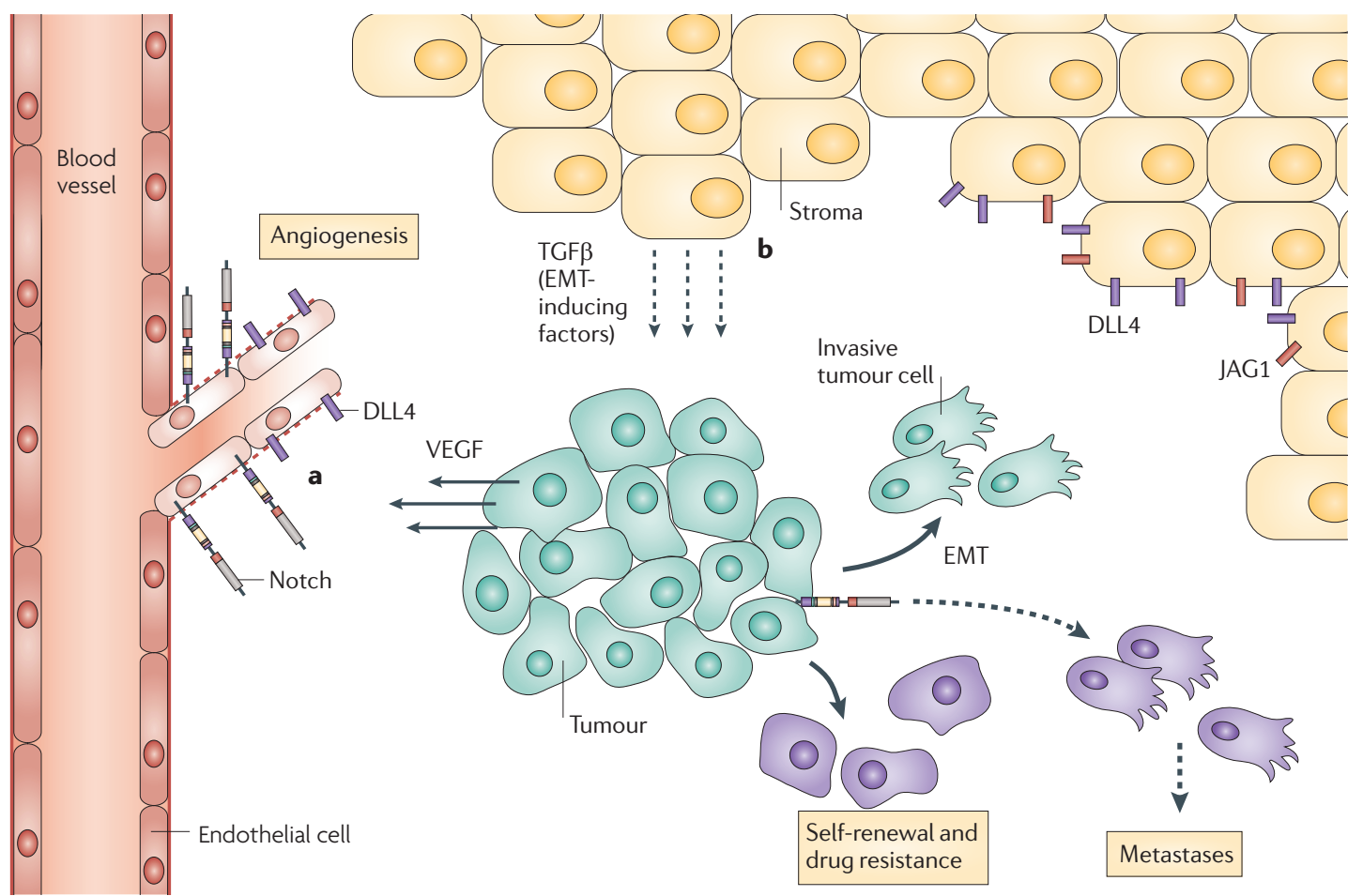

Figure 4 | Notch-regulated tumour-microenvironment interactions in tumour maintenance and progression. a | Notch signalling in angiogenesis is shown. The tumour secretes vascular endothelial growth factor (VEGF), inducing sprouting and branching of new vessels from existing blood vessels. Endothelial tip cells also increase their expression of Delta-like 4 (DLL4; purple) in response to VEGF. DLL4 then signals through Notch on adjacent endothelial tube cells to downregulate the expression of VEGF receptor 2 (VEGFR2) (not shown), leading to the inhibition of angiogenesis. $\mathbf{b}$ | Notch signalling in tumour self-renewal and metastasis is shown. The tumour receives cues from the stroma, including epithelial to mesenchymal transition (EMT)-inducing factors, such as transforming growth factor- $\beta$ (TGF $\beta$ ), in response to which the tumour cells acquire invasive (green invasive cells) or stem-like (purple cells) properties. Some of these cells may acquire both properties (purple invasive cells) (possibly owing to activated Notch signalling) and be able to metastasize and establish secondary tumours. Dashed arrows indicate poorly understood mechanisms.

to chemotherapeutic drugs ${ }^{134}$. In pancreatic cancer, the expression of nuclear NOTCH3 along with phosphoSTAT3 and phospho-AKT is associated with an aggressive tumour phenotype ${ }^{135}$. Inhibiting the Notch pathway also sensitizes otherwise taxane-resistant colon cancer cells to mitotic arrest both in vitro and in vivo, suggesting that combining taxanes with a GSI could be a useful therapeutic strategy ${ }^{136}$.

One mechanism for Notch-induced drug resistance that is evident in pancreatic tumour cell lines is the induction of the transcriptional repressor HES1, which downregulates PTEN in certain cell types ${ }^{137}$. Inhibition of the PI3K survival pathway with wortmannin or LY294002 results in reduced levels of the NICD in prostate cancer cells. This leads to loss of Notch-mediated p53 downregulation and thus sensitization to chemotherapeutic agents ${ }^{138}$. This is further supported by data showing that ectopic expression of NOTCH1 does not confer chemoresistance in cells treated with PI3K inhibitors $^{63}$. Similar effects were observed by blocking mTOR (a kinase acting downstream of PI3K) with rapamycin, which prevents the inhibition of $\mathrm{p} 53$-mediated transcription by Notch, thus sensitizing the cells to drug treatment ${ }^{63}$.
Notch-induced chemoresistance can also result from antagonism between Notch and EGFR, as observed in trastuzumab (Herceptin; Genentech)resistant ERBB2-positive breast cancer. In these tumours, Notch signalling is inactive and the tumours are not sensitive to GSI treatment. However, treatment with trastuzumab or a dual-specificity RTK inhibitor that targets EGFR and ERBB2 induced the upregulation of Notch activity. Treatment with a combination of trastuzumab and a GSI induced apoptosis in these cells ${ }^{139}$.

In oestrogen receptor (ER)-positive breast cancer cells, treatment with tamoxifen inhibits the response to oestrogen, but turns the Notch pathway on, leading to the activation of survival pathways. Notch interacts with ERa at the chromatin level and regulates a subset of ER-dependent genes. This crosstalk is probably dependent on the recruitment of IKKa to the chromatin by Notch, suggesting that IKKa could be a novel therapeutic target to specifically inhibit ER-Notch crosstalk $^{140}$. Interestingly, an important role has been attributed to Notch in the maintenance of ER-negative tumours. These tumours show an increased expression of survivin, increased cell proliferation and reduced 
apoptosis ${ }^{141,142}$. ER-negative tumours show reduced tumour growth when treated with a GSI, indicating a role for Notch pathway in the maintenance of these tumours $^{142}$.

A recent study by Wang et al. ${ }^{143}$ has implicated the Notch pathway in the radioresistance of CSCs. This study demonstrated that inhibiting the Notch pathway with GSIs resulted in a reduction of AKT activity and made the glioma stem cells more radiosensitive ${ }^{143}$. Furthermore, combining GSIs with temozolomide (Temodar; Schering-Plough) treatment blocked the progression of brain tumours in $50 \%$ of the treated mice, which was probably due to blocking Notch in the CSCs and thus sensitizing them to drug treatment ${ }^{144}$.

Taken together, these studies suggest that the activation of the Notch pathway can make tumour cells resistant to chemotherapy or radiation. A deeper understanding of the crosstalk between Notch and other signalling pathways will facilitate the design of novel therapeutic regimens that could sensitize tumour cells to chemotherapeutic agents and radiation.

\section{Conclusions and future directions}

In this Review we have discussed the evidence for a role of aberrant Notch signalling in solid tumours. As the title alludes to, we have found that Notch signalling in solid tumours seems to act in almost every tumorigenic process. Notch activity has been associated with the initiation and progression of neoplastic disease, and has been implicated in the maintenance of the neoplastic phenotype and resistance to therapeutic agents. Surprisingly though, there is little evidence to demonstrate that Notch signalling is constitutively activated through Notch gene mutations in these cancers. In fact, it seems to be likely that the hyperactivation of Notch receptors in tumours is through normal ligand-mediated events and/or loss of negative regulators and, therefore, remains sensitive to GSIs (BOX 3). In fact, there are at least four GSI compounds being evaluated for efficacy in the treatment of various tumours in nearly 20 ongoing clinical trials, which include trials in T-ALL, breast cancer, pancreatic cancer, glioblastoma and melanoma (see ClinicalTrials.gov; see Further information). Furthermore, several novel biological agents (such as, antibodies and decoys) are being developed to inhibit Notch signalling ${ }^{145-147}$. However, evidence also supports a context-dependent role for Notch as a tumour suppressor. Several lines of evidence that have been derived from mouse models suggest that the loss of Notch 1 can promote tumorigenesis. Although Notch itself does not fit the classical definition of a tumour suppressor, the loss of Notch activity can provide the proper environment to promote tumorigenesis in certain contexts. For example, it is possible that the loss of Notch activity could result in a change in cell fate to a cell type with greater proliferative capacity that may then be more prone to transformation.

What accounts for these pleiotropic effects that are governed by Notch signalling? Can we predict the outcome of Notch signalling in any given tumour? Perhaps Notch signalling in tumorigenesis represents a new paradigm in oncogenic signalling pathways. Unlike the 'classical' oncogenes such as RAS isoforms or BRAF, in which mutation renders activity constitutive in all cells, the Notch pathway seems to be inappropriately activated depending on cellular context. Moreover, not all Notch signalling is equal. Evidence suggests that the four Notch proteins have distinct activities and outcomes, although it is thought that the mechanistic details of action are similar. In fact, there is currently no clarity regarding specificity in Notch signalling with respect to each Notch protein. To compound this problem, recent evidence has suggested that distinct populations within a tumour can express distinct Notch paralogues. For example, in breast carcinoma the CSC population displayed NOTCH4 expression and activity, whereas the more differentiated cancer cells expressed NOTCH1 (REF. 148). Blocking NOTCH4 but not NOTCH1 by small interfering RNA negatively affects the CSCs ${ }^{148}$. Furthermore, evidence exists indicating that NOTCH2 can have a role in the progression of pancreatic carcinoma but that NOTCH1 cannot ${ }^{149}$. In fact, NOTCH1 may even have an opposing tumour suppressor function in pancreatic carcinoma ${ }^{5}$. If all four Notch proteins function in a mechanistically similar manner, how can these different activities be explained? Although much work will have to be done to answer these questions, it is intriguing to speculate that the different activities among the Notch proteins are primarily mediated by events on chromatin in the regulation of transcription. If we consider that the Notch-CSL-MAML1 core complex represents the initial scaffold on which a transcriptional regulatory complex is built, one can imagine that this is where the specificity lies. Certainly, we can hypothesize that, considering the milieu of transcriptional regulatory proteins, distinct Notch complexes can recruit or interact with a variety of factors that modulate the transcription of Notch target genes. Considering this concept, it becomes more evident how pathway crosstalk can influence Notch signalling outcome.

This then presents a problem in that many contextual cues via pathway crosstalk might determine the outcome of a cancer treatment that is based on the inhibition of Notch signalling. Thus, the barrier to effective combinatorial treatment regimens will be the elucidation of the relevant signalling networks that interface with Notch. Despite the wealth of studies investigating aspects of Notch signalling, the research field is still lacking the emergence of universal themes that would provide information about how Notch affects so many neoplasms and whether the inhibition of Notch signalling would prove to be a 'magic bullet' in cancer care. However, what we have discovered is that Notch is not the whole story, but merely the preface to a 'Tolstoyesque' epic. Research in the coming years should aim to decipher the complex crosstalk networks that are governed by Notch and that influence Notch signalling. Only then will we be able to effectively target the Notch pathway in cancer. 
1. Ellisen, L. W. et al. TAN-1, the human homolog of the Drosophila notch gene, is broken by chromosomal translocations in T lymphoblastic neoplasms. Cell 66, 649-661 (1991)

NOTCH 1 was found to be affected by the chromosomal translocation $\mathrm{t}(7 ; 9)(\mathrm{q} 34 ; \mathrm{q} 34.3)$ which was found in several patients with T-ALL. This was the first instance in which NOTCH1 was implicated in human cancer.

2. Weng, A. P. et al. Activating mutations of $\mathrm{NOTCH} 1$ in human T cell acute lymphoblastic leukemia. Science 306, 269-271 (2004).

The authors found that more than $50 \%$ of human T-ALLs have activating mutations in NOTCH 1.

3. Malecki, M. J. et al. Leukemia-associated mutations within the NOTCH 1 heterodimerization domain fall into at least two distinct mechanistic classes. Mol. Cell. Biol. 26, 4642-4651 (2006).

4. Koch, U. \& Radtke, F. Notch and cancer: a doubleedged sword. Cell. Mol. Life Sci. 64, 2746-2762 (2007).

5. Hanlon, L. et al. Notch 1 functions as a tumor suppressor in a model of K-ras-induced pancreatic ductal adenocarcinoma. Cancer Res. 70, 4280-4286 (2010).

6. Plentz, R. et al. Inhibition of $\gamma$-secretase activity inhibits tumor progression in a mouse model of pancreatic ductal adenocarcinoma. Gastroenterology 136, 1741-1749 e1746 (2009).

7. Santagata, S. et al. JAGGED1 expression is associated with prostate cancer metastasis and recurrence. Cancer Res. 64, 6854-6857 (2004).

8. Miyamoto, Y. et al. Notch mediates TGF $\alpha$-induced changes in epithelial differentiation during pancreatic tumorigenesis. Cancer Cell 3, 565-576 (2003).

9. Brennan, C. et al. Glioblastoma subclasses can be defined by activity among signal transduction pathways and associated genomic alterations. PLOS ONE 4, e7752 (2009).

10. Reedijk, M. et al. High-level coexpression of JAG1 and NOTCH 1 is observed in human breast cancer and is associated with poor overall survival. Cancer Res. 65 8530-8537 (2005).

11. Aster, J. C., Pear, W. S. \& Blacklow, S. C. Notch signaling in leukemia. Annu. Rev. Pathol. 3, 587-613 (2008).

12. Gordon, W. R., Arnett, K. L. \& Blacklow, S. C. The molecular logic of Notch signaling-a structural and biochemical perspective. J. Cell Sci. 121, 3109-3119 (2008).

13. Bray, S. J. Notch signalling: a simple pathway becomes complex. Nature Rev. Mol. Cell Biol. 7, 678-689 (2006).

14. Kopan, R. \& Ilagan, M. X. The canonical Notch signaling pathway: unfolding the activation mechanism. Cell 137, 216-233 (2009). A comprehensive review on Notch signalling

15. Kovall, R. A. More complicated than it looks: assembly of Notch pathway transcription complexes. Oncogene 27, 5099-5109 (2008)

16. Fortini, M. E. Notch signaling: the core pathway and its posttranslational regulation. $\mathrm{Dev}$. Cell 16 633-647 (2009)

17. LaVoie, M. J. \& Selkoe, D. J. The Notch ligands, Jagged and Delta, are sequentially processed by $\alpha$-secretase and presenilin $/ \gamma$-secretase and release signaling fragments. J. Biol. Chem. 278 , 34427-34437 (2003)

18. Six, E. et al. The Notch ligand Delta 1 is sequentially cleaved by an ADAM protease and $\gamma$-secretase Proc Natl Acad. Sci. USA 100, 7638-7643 (2003).

19. Bland, C. E., Kimberly, P. \& Rand, M. D. Notchinduced proteolysis and nuclear localization of the Delta ligand. J. Biol. Chem. 278, 13607-13610 (2003).

20. Klueg, K. M., Parody, T. R. \& Muskavitch, M. A Complex proteolytic processing acts on Delta, a transmembrane ligand for Notch, during Drosophila development. Mol. Biol. Cell 9, 1709-1723 (1998).

21. D'Souza, B., Meloty-Kapella, L. \& Weinmaster, C. Canonical and non-canonical Notch ligands. Curr. Top. Dev. Biol. 92, 73-129 (2010).

22. Ascano, J. M., Beverly, L. J. \& Capobianco, A. J. The C-terminal PDZ-ligand of JAGGED1 is essential for cellular transformation. J. Biol. Chem. 278 , 8771-8779 (2003). The first evidence suggesting a bidirectional signalling by Notch ligands independently of Notch. The authors demonstrate that JAG1 can transform cells and this requires the PDZ ligand motif of the ICD.
23. Kolev, V. et al. The intracellular domain of Notch ligand Delta 1 induces cell growth arrest. FEBS Lett. 579, 5798-5802 (2005)

24. Gallahan, D., Kozak, C. \& Callahan, R. A new common integration region (int-3) for mouse mammary tumor virus on mouse chromosome 17.J. Virol. 61 218-220 (1987).

25. Gallahan, D. \& Callahan, R. The mouse mammary tumor associated gene INT3 is a unique member of the NOTCH gene family (NOTCH4). Oncogene 14, 1883-1890 (1997).

This study, together with reference 24 , identified the Notch locus as a common integration site in MMTV-induced tumours. Subsequently identified as NOTCH4, it was suggested that this has a role in mammary tumorigenesis.

26. Gallahan, D. et al. Expression of a truncated Int3 gene in developing secretory mammary epithelium specifically retards lobular differentiation resulting in tumorigenesis. Cancer Res. 56, 1775-1785 (1996). Expression of a truncated form of the Int3 (Notch4) gene in the mammary epithelium of mice resulted in highly malignant mammary tumours and lung metastases.

27. Capobianco, A. J., Zagouras, P., Blaumueller, C. M Artavanis-Tsakonas, S. \& Bishop, J. M. Neoplastic transformation by truncated alleles of human NOTCH1/TAN1 and NOTCH2. Mol. Cell. Biol. 17, 6265-6273 (1997)

A constitutively active truncated form of NOTCH 1 and NOTCH2, in cooperation with the E1 A adenovirus oncogene, can transform RKE cells.

28. Demarest, R. M., Ratti, F. \& Capobianco, A. J. It's T-ALL about Notch. Oncogene 27, 5082-5091 (2008)

29. Ronchini, C. \& Capobianco, A. J. Induction of cyclin D1 transcription and CDK2 activity by Notch(ic): implication for cell cycle disruption in transformation by Notch(ic). Mol. Cell. Biol. 21, 5925-5934 (2001).

30. Ling, H., Sylvestre, J. R. \& Jolicoeur, P. Notch 1-induced mammary tumor development is cyclin D1-dependent and correlates with expansion of pre-malignant multipotent duct-limited progenitors. Oncogene 29, 4543-4554 (2010)

31. Cohen, B. et al. Cyclin D1 is a direct target of JAG1-mediated Notch signaling in breast cancer. Breast Cancer Res. Treat. 123, 113-124 (2010).

32. Sicinska, E. et al. Requirement for cyclin D3 in lymphocyte development and T cell leukemias. Cancer Cell 4, 451-461 (2003).

33. Sharma, V. M. et al. Notch1 contributes to mouse T-cell leukemia by directly inducing the expression of c-myc. Mol. Cell. Biol. 26, 8022-8031 (2006).

34. Weng, A. P. et al. c-Myc is an important direct target of Notch 1 in T-cell acute lymphoblastic leukemia/ lymphoma. Genes Dev. 20, 2096-2109 (2006).

35. Klinakis, A. et al. Myc is a Notch 1 transcriptional target and a requisite for Notch 1 -induced mammary tumorigenesis in mice. Proc. Natl Acad. Sci. USA 103, 9262-9267 (2006).

36 Palomero, T. et al. NOTCH1 directly regulates c-MYC and activates a feed-forward-loop transcriptional network promoting leukemic cell growth. Proc. Natl Acad. Sci. USA 103, 18261-18266 (2006).

37. Murata, J. et al. Notch-Hes1 pathway contributes to the cochlear prosensory formation potentially through the transcriptional down-regulation of p27Kip 1 . J. Neurosci. Res. 87, 3521-3534 (2009).

38. Monahan, P., Rybak, S. \& Raetzman, L. T. The notch target gene HES1 regulates cell cycle inhibitor expression in the developing pituitary. Endocrinology 150, 4386-4394 (2009).

39. Riccio, O. et al. Loss of intestinal crypt progenitor cells owing to inactivation of both Notch1 and Notch2 is accompanied by derepression of CDK inhibitors p27Kip1 and p57Kip2. EMBO Rep. 9, 377-383 (2008)

40. Dohda, T. et al. Notch signaling induces SKP2 expression and promotes reduction of p27Kip 1 in T-cell acute lymphoblastic leukemia cell lines. Exp. Cell Res. 313, 3141-3152 (2007).

41. Mittal, S., Subramanyam, D., Dey, D., Kumar, R. ¿ Rangarajan, A. Cooperation of Notch and Ras/MAPK signaling pathways in human breast carcinogenesis. Mol. Cancer 8, 128 (2009).

42. Xu, P. et al. The oncogenic roles of Notch 1 in astrocytic gliomas in vitro and in vivo. J. Neurooncol. 97, 41-51 (2010)

43. Liu, W., Singh, S. R. \& Hou, S. X. JAK-STAT is restrained by Notch to control cell proliferation of the Drosophila intestinal stem cells. J. Cell. Biochem. 109 992-999 (2010).
44. Li, T. et al. Epidermal growth factor receptor and notch pathways participate in the tumor suppressor function of $\gamma$-secretase. J. Biol. Chem. 282, 32264-32273 (2007).

45. Lefort, K. et al. Notch1 is a p53 target gene involved in human keratinocyte tumor suppression through negative regulation of ROCK $1 / 2$ and MRCKa kinases. Genes Dev. 21, 562-577 (2007)

46. Wang, C. et al. Notch 1 signaling sensitizes tumor necrosis factor-related apoptosis-inducing ligandinduced apoptosis in human hepatocellular carcinoma cells by inhibiting Akt/Hdm2-mediated $\mathrm{p} 53$ degradation and up-regulating p53-dependent DR5 expression. J. Biol. Chem. 284, 16183-16190 (2009).

This study suggests a mechanism by which Notch signalling induces apoptosis by activating p53.

47. Wang, L. et al. Overexpressed active Notch 1 induces cell growth arrest of HeLa cervical carcinoma cells. Int J. Gynecol. Cancer 17, 1283-1292 (2007).

48. Talora, C. et al. Constitutively active Notch 1 induces growth arrest of HPV-positive cervical cancer cells via separate signaling pathways. Exp. Cell Res. 305, 343-354 (2005)

49. Dotto, G. P. Crosstalk of Notch with $p 53$ and $p 63$ in cancer growth control. Nature Rev. Cancer 9 587-595 (2009) A comprehensive review on the crosstalk between Notch and p53, which probably determines whether Notch is oncogenic or tumour suppressive.

50. Shou, J., Ross, S., Koeppen, H., de Sauvage, F. J. \& Gao, W. Q. Dynamics of notch expression during murine prostate development and tumorigenesis. Cancer Res. 61, 7291-7297 (2001).

51. Whelan, J. T., Kellogg, A., Shewchuk, B. M., Hewan-Lowe, K. \& Bertrand, F. E. Notch-1 signaling is lost in prostate adenocarcinoma and promotes PTEN gene expression. J. Cell. Biochem. 107, 992-1001 (2009).

52. Bin Hafeez, B. et al. Targeted knockdown of Notch1 inhibits invasion of human prostate cancer cells concomitant with inhibition of matrix metalloproteinase- 9 and urokinase plasminogen activator. Clin. Cancer Res. 15, 452-459 (2009).

53. Niimi, H., Pardali, K., Vanlandewijck, M., Heldin, C. H. $\&$ Moustakas, A. Notch signaling is necessary for epithelial growth arrest by TGF- $\beta$. J. Cell Biol. 176 695-707 (2007)

54. Sun, Y. et al. Notch4 intracellular domain binding to Smad3 and inhibition of the TGF- $\beta$ signaling. Oncogene 24, 5365-5374 (2005).

55. Masuda, S. et al. Notch1 oncoprotein antagonizes TGF- $\beta /$ Smad-mediated cell growth suppression via sequestration of coactivator p300. Cancer Sci. 96 , 274-282 (2005)

56. Demehri, S., Turkoz, A. \& Kopan, R. Epidermal Notch loss promotes skin tumorigenesis by impacting the stromal microenvironment. Cancer Cell 16, 55-66 (2009).

This study demonstrates the role of the microenvironment in determining the role of Notch signalling in the skin, where Notch is known to have a tumour suppressor effect. The study shows that intact Notch signalling is not sufficient to prevent tumorigenesis when the microenvironment is tumour promoting.

57. Dumortier, A. et al. Atopic dermatitis-like disease and associated lethal myeloproliferative disorder arise from loss of Notch signaling in the murine skin. PLOS ONE 5, e9258 (2010).

58. Demehri, S. et al. Notch-deficient skin induces a lethal systemic B-lymphoproliferative disorder by secreting TSLP, a sentinel for epidermal integrity. PLOS Biol. 6 , e123 (2008)

59. Palomero, T. Dominguez, M. \& Ferrando, A. A. The role of the PTEN/AKT Pathway in NOTCH1-induced leukemia. Cell Cycle 7, 965-970 (2008)

60. Nair, P., Somasundaram, K. \& Krishna, S. Activated Notch 1 inhibits p53-induced apoptosis and sustains transformation by human papillomavirus type $16 \mathrm{E} 6$ and E7 oncogenes through a PI3K-PKB/Aktdependent pathway. J. Virol. 77, 7106-7112 (2003).

61. Meurette, O. et al. Notch activation induces Akt signaling via an autocrine loop to prevent apoptosis in breast epithelial cells. Cancer Res. 69, 5015-5022 (2009).

62. Wendorff, A. A. et al. Hes 1 is a critical but contextdependent mediator of canonical Notch signaling in lymphocyte development and transformation. Immunity 33, 671-684 (2010). 
63. Mungamuri, S., Yang, X., Thor, A. \& Somasundaram, K. Survival signaling by Notch 1 : mammalian target of rapamycin (mTOR)-dependent inhibition of p53 Cancer Res. 66, 4715-4724 (2006).

64. Beverly, L., Felsher, D. \& Capobianco, A. Suppression of p53 by Notch in lymphomagenesis: implications for initiation and regression. Cancer Res. 65, 7159-7168 (2005).

A very elegant tetracycline-inducible mouse model, Top-Notch ${ }^{\text {ic }}$, was used to show that Notch suppresses p53 through repression of the ARF-MDM2-p53 tumour surveillance pathway in lymphomagenesis.

65. Song, L. L. et al. Notch-1 associates with IKKa and regulates IKK activity in cervical cancer cells. Oncogene 27, 5833-5844 (2008)

66. Ramdass, B. et al. Coexpression of Notch 1 and NF-kB signaling pathway components in human cervical cancer progression. Gynecol. Oncol. 104, 352-36 (2007).

67. Osipo, C., Golde, T. E., Osborne, B. A. \& Miele, L. A. Off the beaten pathway: the complex cross talk between Notch and NF-kB. Lab. Invest. 88, 11-17 (2008).

68. Nakamura, T., Tsuchiya, K. \& Watanabe, M. Crosstalk between Wnt and Notch signaling in intestinal epithelial cell fate decision. J. Gastroenterol. 42, 705-710 (2007)

69. Bienz, M. \& Clevers, H. Linking colorectal cancer to Wnt signaling. Cell 103, 311-320 (2000)

70. Inomata, M., Ochiai, A., Akimoto, S., Kitano, S. \& Hirohashi, S. Alteration of $\beta$-catenin expression in colonic epithelial cells of familial adenomatous polyposis patients. Cancer Res. 56, 2213-2217 (1996).

71. Satoh, S. et al. AXIN1 mutations in hepatocellular carcinomas, and growth suppression in cancer cells by virus-mediated transfer of AXIN1. Nature Genet. 24 245-250 (2000)

72. Korinek, V. et al. Constitutive transcriptional activation by a $\beta$-catenin-Tcf complex in APC ${ }^{-1}$ colon carcinoma. Science 275, 1784-1787 (1997).

73. Caldwell, G. et al. The Wnt antagonist sFRP1 in colorectal tumorigenesis. Cancer Res. 64, 883-888 (2004).

74. Suzuki, H. et al. Epigenetic inactivation of SFRP genes allows constitutive WNT signaling in colorectal cancer. Nature Genet. 36, 417-422 (2004).

75. Katoh, M., Kirikoshi, H., Terasaki, H. \& Shiokawa, K WNT2B2 mRNA, up-regulated in primary gastric cancer, is a positive regulator of the WNT$\beta$-catenin-TCF signaling pathway. Biochem. Biophys. Res. Commun. 289, 1093-1098 (2001).

76. Kirikoshi, H., Sekihara, H. \& Katoh, M. Expression of WNT14 and WNT14B mRNAs in human cancer, up-regulation of WNT 14 by IFN $\gamma$ and up-regulation of WNT14B by $\beta$-estradiol. Int. J. Oncol. 19 , $1221-1225$ (2001).

77. Okino, K. et al. Up-regulation and overproduction of DVL-1, the human counterpart of the Drosophila dishevelled gene, in cervical squamous cell carcinoma. Oncol. Rep. 10, 1219-1223 (2003).

78. Terasaki, H., Saitoh, T., Shiokawa, K. \& Katoh, M Frizzled-10, up-regulated in primary colorectal cancer, is a positive regulator of the WNT - $\beta$-catenin - TCF signaling pathway. Int. J. Mol. Med. 9, 107-112 (2002).

79. Bjorklund, P., Akerstrom, G. \& Westin, G. An LRP5 receptor with internal deletion in hyperparathyroid tumors with implications for deregulated WNT/ß-catenin signaling. PLoS Med. 4, e328 (2007).

80. van Es, J. et al. Notch $/ \gamma$-secretase inhibition turns proliferative cells in intestinal crypts and adenomas into goblet cells. Nature 435, 959-963 (2005).

81. Axelrod, J., Matsuno, K., Artavanis-Tsakonas, S. \& Perrimon, N. Interaction between Wingless and Notch signaling pathways mediated by dishevelled. Science 271, 1826-1832 (1996). D. melanogaster DSH, which is a transducer of WNT signalling, antagonizes the Notch pathway through a direct physical interaction with the C terminus of Notch.

82. Couso, J. \& Martinez Arias, A. Notch is required for wingless signaling in the epidermis of Drosophila. Cell 79, 259-272 (1994)

83. Giraldez, A. \& Cohen, S. Wingless and Notch signaling provide cell survival cues and control cell proliferation during wing development. Development 130 , 6533-6543 (2003).

84. Jin, Y. et al. $\beta$-catenin modulates the level and transcriptional activity of Notch $1 /$ NICD through its direct interaction. Biochim. Biophys. Acta 1793, 290-299 (2009)
85. Alves-Guerra, M Ronchini, C \& Capobianco, A Mastermind-like 1 is a specific coactivator of $\beta$-catenin transcription activation and is essential for colon carcinoma cell survival. Cancer Res. 67, 8690-8698 (2007).

This paper is the first report of a role for MAML1 outside of the Notch pathway. MAML1 is recruited to the CCND1 promoter by $\beta$-catenin, and knock down of MAML1 in colonic carcinoma cells results in cell death.

86. Nicolas, M. et al. Notch 1 functions as a tumor suppressor in mouse skin. Nature Genet. 33 , 416-421 (2003).

A key paper demonstrating a tumour suppressor role for NOTCH 1 and also the interactions with the WNT pathway.

87. Devgan, V., Mammucari, C., Millar, S. E., Brisken, C. \& Dotto, G. P. p21WAF1/Cip1 is a negative transcriptional regulator of Wnt4 expression downstream of Notch 1 activation. Genes Dev. 19 1485-1495 (2005)

88. De La, O. J. et al. Notch and Kras reprogram pancreatic acinar cells to ductal intraepithelia neoplasia. Proc. Natl Acad. Sci. USA 105 18907-18912 (2008)

89. De La, O J \& Murtaugh, L. C. Notch and Kras in pancreatic cancer: at the crossroads of mutation, differentiation and signaling. Cell Cycle 8 1860-1864 (2009).

90. Weiizen, S. et al. Activation of Notch-1 signaling maintains the neoplastic phenotype in human Rastransformed cells. Nature Med. 8, 979-986 (2002). A study that gives an insight into the role of Notch signalling in RAS-driven cancers.

91. Claxton, S. \& Fruttiger, M. Periodic Delta-like 4 expression in developing retinal arteries. Gene Expr. Patterns 5, 123-127 (2004).

92. Hellstrom, M. et al. DII4 signalling through Notch 1 regulates formation of tip cells during angiogenesis. Nature 445, 776-780 (2007).

93. Suchting, S. et al. The Notch ligand Delta-like 4 negatively regulates endothelial tip cell formation and vessel branching. Proc. Natl Acad. Sci. USA 104 3225-3230 (2007)

94. Mailhos, C et al. Delta 4, an endothelial specific notch ligand expressed at sites of physiological and tumor angiogenesis. Differentiation 69, 135-144 (2001)

95. Patel, N. et al. Up-regulation of endothelial delta-like 4 expression correlates with vessel maturation in bladder cancer. Clin. Cancer Res. 12, 4836-4844 (2006).

96. Thurston, G., Noguera-Troise, I. \& Yancopoulos, G. The Delta paradox: DLL4 blockade leads to more tumour vessels but less tumour growth. Nature Rev. Cancer 7 327-331 (2007)

97. Patel, N et al. Up-regulation of delta-like 4 ligand in human tumor vasculature and the role of basal expression in endothelial cell function. Cancer Res. 65, 8690-8697 (2005)

98. Noguera-Troise, I. et al. Blockade of DIl4 inhibits tumour growth by promoting non-productive angiogenesis. Nature 444, 1032-1037 (2006).

99. Benedito, R. et al. The notch ligands DII4 and Jagged 1 have opposing effects on angiogenesis. Cell 137 1124-1135 (2009).

\section{ifferential effects of Notch ligands on}

angiogenesis. The study suggests that modification by Fringe probably modulates Notch activity during angiogenesis.

100. Panin, V. M., Papayannopoulos, V., Wilson, R. \& Irvine, K. D. Fringe modulates Notch-ligand interactions. Nature 387, 908-912 (1997).

101. Fu, Y. et al. Differential regulation of transforming growth factor $\beta$ signaling pathways by Notch in human endothelial cells. J. Biol. Chem. 284, 19452-19462 (2009).

102. Itoh, F. et al. Synergy and antagonism between Notch and BMP receptor signaling pathways in endothelial cells. EMBO J. 23, 541-551 (2004).

103. Thiery, J. P. Epithelial-mesenchymal transitions in development and pathologies. Curr. Opin. Cell Biol. 15, 740-746 (2003). A comprehensive review on EMT

104. Leong, K. et al. Jagged 1-mediated Notch activation induces epithelial-to-mesenchymal transition throug Slug-induced repression of E-cadherin. J. Exp. Med. 204, 2935-2948 (2007).

105. Chen, J., Imanaka, N. \& Griffin, J. D. Hypoxia potentiates Notch signaling in breast cancer leading to decreased E-cadherin expression and increased cell migration and invasion. Br. J. Cancer 102, 351-360 (2010)
106. Sahlgren, C. Gustafsson, M. V., Jin, S., Poellinger L. \& Lendahl, U. Notch signaling mediates hypoxia-induced tumor cell migration and invasion. Proc. Natl Acad. Sci. USA 105, 6392-6397 (2008).

107. Zavadil, J., Cermak, L., Soto-Nieves, N. \& Bottinger, E. P. Integration of TGF- $\beta /$ Smad and Jagged $1 /$ Notch signalling in epithelial-to-mesenchymal transition. EMBO J. 23, 1155-1165 (2004)

108. Mani, S. et al. The epithelial-mesenchymal transition generates cells with properties of stem cells. Cell 133 704-715 (2008)

This paper demonstrates that the induction of an EMT in immortalized mammary epithelial cells results in the expression of stem cell markers and an increased ability to self-renew. The paper suggests EMT as a mechanism of conferring stem-like properties to cells.

109. Lapidot, T. et al. A cell initiating human acute myeloid leukaemia after transplantation into SCID mice. Nature 367, 645-648 (1994).

110. Bonnet, D. \& Dick, J. Human acute myeloid leukemia is organized as a hierarchy that originates from a primitive hematopoietic cell. Nature Med. 3, 730-737 (1997).

111. Cicalese, A. et al. The tumor suppressor p53 regulates polarity of self-renewing divisions in mammary stem cells. Cell 138, 1083-1095 (2009).

112. Al-Hajj, M., Wicha, M., Benito-Hernandez, A. Morrison, S. \& Clarke, M. Prospective identification of tumorigenic breast cancer cells. Proc. Natl Acad. Sci. USA 100, 3983-3988 (2003).

113. Boiko, A. D. et al. Human melanoma-initiating cells express neural crest nerve growth factor receptor CD271. Nature 466, 133-137 (2010).

114. Lee, C., Dosch, J. \& Simeone, D. Pancreatic cancer stem cells. J. Clin. Oncol. 26, 2806-2812 (2008).

115. Maitland, N. \& Collins, A. Prostate cancer stem cells: a new target for therapy. J. Clin. Oncol. 26, 2862-2870 (2008)

116. O’Brien, C., Pollett, A., Gallinger, S. \& Dick, J. A human colon cancer cell capable of initiating tumour growth in immunodeficient mice. Nature $\mathbf{4 4 5}$ 106-110 (2007).

117. Peacock, C \& Watkins, D. Cancer stem cells and the ontogeny of lung cancer. J. Clin. Oncol. 26 , 2883-2889 (2008).

118. Prince, M. \& Ailles, L. Cancer stem cells in head and neck squamous cell cancer. J. Clin. Oncol. 26 2871-2875 (2008)

119. Sell, S. \& Leffert, H. Liver cancer stem cells. J. Clin. Oncol. 26, 2800-2805 (2008)

120. Singh, S. et al. Identification of a cancer stem cell in human brain tumors. Cancer Res. 63, 5821-5828 (2003).

121. Bao, S et al. Glioma stem cells promote radioresistance by preferential activation of the DNA damage response. Nature 444, 756-760 (2006)

122. Dean, M., Fojo, T. \& Bates, S. Tumour stem cells and drug resistance. Nature Rev. Cancer 5, 275-284 (2005).

123. Bao, S. et al. Stem cell-like glioma cells promote tumor angiogenesis through vascular endothelia growth factor. Cancer Res. 66, 7843-7848 (2006)

124. Pistollato, F. et al. Interaction of hypoxia-inducible factor- $1 \alpha$ and Notch signaling regulates medulloblastoma precursor proliferation and fate. Stem Cells 28, 1918-1929 (2010).

125. Varnum-Finney, B. et al. Pluripotent, cytokine dependent, hematopoietic stem cells are immortalized by constitutive Notch 1 signaling. Nature Med. 6 , 1278-1281 (2000)

126. Dontu, G. et al. Role of Notch signaling in cell-fate determination of human mammary stem/progenitor cells. Breast Cancer Res. 6, R605-R615 (2004).

127. Farnie, G. \& Clarke, R. Mammary stem cells and breast cancer-role of Notch signalling. Stem Cell Rev. 3, 169-175 (2007)

128. Farnie, G. et al. Novel cell culture technique for primary ductal carcinoma in situ: role of Notch and epidermal growth factor receptor signaling pathways. J. Natl Cancer Inst. 99, 616-627 (2007).

29. Zhang X et al. Notch activation promotes cell proliferation and the formation of neural stem cell-like colonies in human glioma cells. Mol. Cell. Biochem. 307, 101-108 (2008)

130. Hitoshi, S. et al. Notch pathway molecules are essential for the maintenance, but not the generation, of mammalian neural stem cells. Genes Dev. 16 , 846-858 (2002). 
131. Hitoshi, S. et al. Primitive neural stem cells from the mammalian epiblast differentiate to definitive neural stem cells under the control of Notch signaling. Genes Dev. 18, 1806-1811 (2004).

132. Nakamura, Y. et al. The bHLH gene hes 1 as a repressor of the neuronal commitment of CNS stem cells. J. Neurosci. 20, 283-293 (2000).

133. Fan, X. et al. Notch pathway blockade depletes CD 133-positive glioblastoma cells and inhibits growth of tumor neurospheres and xenografts. Stem Cells $\mathbf{2 8}$ 5-16 (2009).

134. Meng, R. D. et al. $\gamma$-secretase inhibitors abrogate oxaliplatin-induced activation of the Notch-1 signaling pathway in colon cancer cells resulting in enhanced chemosensitivity. Cancer Res. 69, 573-582 (2009).

135. Doucas, H. et al. Expression of nuclear Notch3 in pancreatic adenocarcinomas is associated with adverse clinical features, and correlates with the expression of STAT3 and phosphorylated Akt. J. Surg. Oncol. 97, 63-68 (2008)

136. Akiyoshi, T. et al. $\gamma$-secretase inhibitors enhance taxane-induced mitotic arrest and apoptosis in colon cancer cells. Gastroenterology 134, 131-144 (2008)

137. Ristorcelli, E., Beraud, E., Mathieu, S., Lombardo, D. $\&$ Verine, A. Essential role of Notch signaling in apoptosis of human pancreatic tumoral cells mediated by exosomal nanoparticles. Int. J. Cancer 125 , 1016-1026 (2009).

138. Wang, Z. et al. Down-regulation of Notch-1 and Jagged-1 inhibits prostate cancer cell growth, migration and invasion, and induces apoptosis via inactivation of Akt, mTOR, and NF-kB signaling pathways. J. Cell. Biochem. 109, 726-736 (2010).

139. Osipo, C. et al. ErbB-2 inhibition activates Notch-1 and sensitizes breast cancer cells to a $\gamma$-secretase inhibitor. Oncogene 27, 5019-5032 (2008).

140. Hao, L. et al. Notch-1 activates estrogen receptor- $\alpha$-dependent transcription via IKKa in breast cancer cells. Oncogene 29, 201-213 (2010).

141. Lee, C. W. et al. A functional Notch-survivin gene signature in basal breast cancer. Breast Cancer Res. 10, R97 (2008).

142. Lee, C. W., Raskett, C. M., Prudovsky, I. \& Altieri, D. C. Molecular dependence of estrogen receptor-negative breast cancer on a notch-survivin signaling axis. Cancer Res. 68, 5273-5281 (2008)

143. Wang, J. et al. Notch promotes radioresistance of glioma stem cells. Stem Cells 28, 17-28 (2010). Glioma stem cells are resistant to radiation and this paper shows that blocking Notch signalling with GSIs sensitizes these cells to radiation and impairs xenograft formation. The mechanism is thought to be through the reduction of AKT activity.

144. Gilbert, C. A., Daou, M. C. Moser R. P. \& Ross, A. H. $\gamma$-secretase inhibitors enhance temozolomide treatment of human gliomas by inhibiting neurosphere repopulation and xenograft recurrence. Cancer Res. 70, 6870-6879 (2010)

This study, together with references 139 and 142 , demonstrates that Notch signalling is responsible for resistance to chemotherapeutic agents and shows the importance of using other therapeutic agents in combination with Notch inhibitors (GSI) for effective therapy.

145. Dikic, I. \& Schmidt, M. H. Notch: implications of endogenous inhibitors for therapy. Bioessays 32, 481-487 (2010)

146. Wu, Y. et al. Therapeutic antibody targeting of individual Notch receptors. Nature 464, 1052-1057 (2010).

147. Yin, L., Velazquez, O. C. \& Liu, Z. J. Notch signaling: emerging molecular targets for cancer therapy. Biochem. Pharmacol. 80, 690-701 (2010).

148. Harrison, H. et al. Regulation of breast cancer stem cell activity by signaling through the Notch4 receptor. Cancer Res. 70, 709-718 (2010).

149. Mazur, P. K. et al. Notch2 is required for progression of pancreatic intraepithelial neoplasia and development of pancreatic ductal adenocarcinoma. Proc. Natl Acad. Sci. USA 107, 13438-13443 (2010).

150. Tonon, G. et al. $\mathrm{t}(11 ; 19)(\mathrm{q} 21 ; \mathrm{p} 13)$ translocation in mucoepidermoid carcinoma creates a novel fusion product that disrupts a Notch signaling pathway. Nature Genet. 33, 208-213 (2003).

151. Enlund, F. et al. Altered Notch signaling resulting from expression of a WAMTP1-MAML2 gene fusion in mucoepidermoid carcinomas and benign Warthin's tumors. Exp. Cell Res. 292, 21-28 (2004).

152. Lennerz, J. K., Perry, A., Mills, J. C., Huettner, P. C. \& Pfeifer, J. D. Mucoepidermoid carcinoma of the cervix: another tumor with the $\mathrm{t}(11 ; 19)$-associated CRTC1-MAML2 gene fusion. Am. J. Surg. Pathol. 33 835-843 (2009).

153. Behboudi, A. et al. Clear cell hidradenoma of the skin-a third tumor type with a $t(11 ; 19)$-associated TORC1-MAML2 gene fusion. Genes Chromosomes Cancer 43, 202-205 (2005).

154. Martinez, A. M. et al. Polyhomeotic has a tumo suppressor activity mediated by repression of Notch signaling. Nature Genet. 41, 1076-1082 (2009)

155. Ferres-Marco, D. et al. Epigenetic silencers and Notch collaborate to promote malignant tumours by $\mathrm{Rb}$ silencing. Nature 439, 430-436 (2006)

156. Moshkin, Y. M. et al. Histone chaperones ASF and NAP 1 differentially modulate removal of active histone marks by LID-RPD3 complexes during NOTCH silencing. Mol. Cell 35, 782-793 (2009)

157. Goodfellow, H. et al. Gene-specific targeting of the histone chaperone Asf1 to mediate silencing. Dev. Cell 13, 593-600 (2007).

158. Takeuchi, T., Adachi, Y. \& Ohtsuki, Y. Skeletrophin, a novel ubiquitin ligase to the intracellular region of Jagged-2, is aberrantly expressed in multiple myeloma. Am. J. Pathol. 166, 1817-1826 (2005).

159. Takeuchi, T., Adachi, Y., Sonobe, H., Furihata, M. \& Ohtsuki, Y. A ubiquitin ligase, skeletrophin, is a negative regulator of melanoma invasion. Oncogene 25, 7059-7069 (2006).

160. Wu, G. et al. SEL-10 is an inhibitor of notch signaling that targets notch for ubiquitin-mediated protein degradation. Mol Cell. Biol. 21, 7403-7415 (2001).

161. Akhoondi, S. et al. FBXW7/hCDC4 is a general tumor suppressor in human cancer. Cancer Res. 67 9006-9012 (2007).

162. Miyaki, M. et al. Somatic mutations of the CDC4 (FBXW7) gene in hereditary colorectal tumors. Oncology 76, 430-434 (2009).

163. Lan, K. et al. Kaposi's sarcoma herpesvirus-encoded latency-associated nuclear antigen stabilizes intracellular activated Notch by targeting the Sel10 protein. Proc. Natl Acad. Sci. USA 104, 16287-16292 (2007).

164. McGill, M. A. \& McGlade, C. J. Mammalian numb proteins promote Notch 1 receptor ubiquitination and degradation of the Notch 1 intracellular domain. J. Biol. Chem. 278, 23196-23203 (2003).

165. Pece, S. et al. Loss of negative regulation by Numb over Notch is relevant to human breast carcinogenesis. J. Cell Biol. 167, 215-221 (2004).

166. Colaluca, I. N. et al. NUMB controls p53 tumour suppressor activity. Nature 451, 76-80 (2008).

167. Matsuno, K., Diederich, R. J., Go, M. J., Blaumueller, C. M. \& Artavanis-Tsakonas, S. Deltex acts as a positive regulator of Notch signaling through interactions with the Notch ankyrin repeats. Development 121, 2633-2644 (1995).

168. Matsuno, K. et al. Human deltex is a conserved regulator of Notch signalling. Nature Genet. 19, 74-78 (1998).

169. Izon, D. J. et al. Deltex 1 redirects lymphoid progenitors to the $\mathrm{B}$ cell lineage by antagonizing Notch 1. Immunity 16, 231-243 (2002).

170. Yamamoto, N. et al. Role of Deltex-1 as a transcriptional regulator downstream of the Notch receptor. J. Biol. Chem. 276, 45031-45040 (2001).

\section{Acknowledgements}

The authors would like to thank members of the Capobianco laboratory for support and D. Robbins and J. Diez for critical reading of the manuscript and useful suggestions. Work in the authors' laboratory is partly supported by grants from the US National Cancer Institute (ROI CA 83736) and the Samuel Waxman Foundation for Cancer Research, USA. The authors sincerely apologize to all their colleagues whose work has not been referenced in this article.

Competing interests statement

The authors declare no competing financial interests.

FURTHER INFORMATION

Anthony J. Capobianco's homepage: http://biomed.miami edu/capobiancolab

ClinicalTrials.gov: http://clinicaltrials.gov

SUPPLEMENTARY INFORMATION

See online article: $\underline{\mathrm{S} 1}$ (table)

ALL LINKS ARE ACTIVE IN THE ONLINE PDF 